\title{
An optimal sensor placement strategy for reliable expansion of mode shapes under measurement noise and modelling error
}

\author{
Mahesh Murugan Jaya ${ }^{1 *}$, Rosario Ceravolo ${ }^{2}$, Luca Zanotti Fragonara ${ }^{3}$ and Emiliano Matta ${ }^{4}$
}

${ }^{1} \mathrm{PhD}$ Student, Department of Structural and Geotechnical Engineering, Politecnico di Torino, Torino, Italy.

${ }^{2}$ Professor, Department of Structural and Geotechnical Engineering, Politecnico di Torino, Torino, Italy.

${ }^{3}$ Lecturer, Centre for Autonomous and Cyberphysical Systems, Cranfield University, Cranfield, United Kingdom.

${ }^{4}$ Assistant Professor, Department of Architecture and Design, Politecnico di Torino, Torino, Italy.

\begin{abstract}
Modal expansion techniques are typically used to expand the experimental modal displacements at sensor positions to all unmeasured degrees of freedom. Since in most cases, sensors can be attached only at limited locations in a structure, an expansion is essential to determine mode shapes, strains, stresses, etc. throughout the structure which can be used for structural health monitoring. Conventional sensor placement algorithms are mostly aimed to make the modal displacements at sensor positions of different modes as linearly independent as possible. However, under the presence of modelling errors and measurement noise, an optimal location based on this criterion is not guaranteed to provide an expanded mode shape which is close to the real mode shape. In this work, the expected value of normal distance between the real mode shape and the expanded mode shape is used as a measure of closeness between the two entities. Optimal sensor locations can be determined by minimizing this distance. This new criterion is applied on a simple cantilever beam and an industrial milling tower. In both cases, by using an exhaustive search of all possible sensor configurations it was possible to find sensor locations which resulted in a significant reduction in the distance when compared to a conventional optimal sensor placement strategy. Sufficiently accurate sub-optimal sequential sensor placement algorithm is also suggested as an alternative to the exhaustive search which is then compared with a genetic algorithmbased search. The efficiency of this new sensor placement criterion is further verified using Monte Carlo simulations for some realistic modelling error conditions.
\end{abstract}

Keywords: Optimal sensor placement; structural health monitoring; operational modal analysis; industrial structures

${ }^{*}$ Corresponding author.

E-mail addresses: mahesh.murugan@polito.it, $\quad$ rosario.ceravolo@polito.it,

1.zanottifragonara@cranfield.ac.uk, emiliano.matta@polito.it 


\section{List of symbols}

Ф Numerical mode shape

$\boldsymbol{\varphi}$ Real mode shape

$\Psi \quad$ Experimental mode shape at sensor locations

$\Psi \quad$ Expanded experimental mode shape

C Transformation matrix for modal expansion

S Sensor configuration

$\mathbf{I}_{\boldsymbol{n}} \quad$ Identity matrix of size $n$

$\boldsymbol{\varepsilon} \quad$ Modelling error in mode shape

$\eta \quad$ Measurement noise in mode shape

$\mathbb{R} \quad$ Set of real numbers

$\operatorname{Tr}(\mathbf{A}) \quad$ Trace of a square matrix $\mathbf{A}$

$\|\mathbf{X}\|_{2} \quad$ Sum of squares of all elements of vector $\mathbf{X}$

$\mathrm{C}_{\mathrm{s}}^{\mathrm{r}} \quad$ Number of $s$ combinations from $r$ when the order is not important

MSE Mean square error

MAC Modal Assurance Criterion matrix

dof Degrees of freedom

$n \quad$ Total number of degrees of freedom

$s \quad$ Number of sensors used

$r \quad$ Possible number of sensor positions

$\sigma \quad$ Standard deviation of a probability density function

$\mu \quad$ Mean of a probability density function

\section{Introduction}

Whether in conducting modal analysis tests or for structural health monitoring, a strategy for placement of sensors is of vital importance. The number of sensors which can be employed in practice is limited by factors such as cost, availability of power, accessibility of the structure, etc. Hence, the sensors which are deployed should be placed such that they maximise their intended utility. Mode shape is an important structural characteristic to be estimated for all modal analysis tests and in most health monitoring systems.

Based on the modal displacements evaluated at the sparse sensor positions, it may be required in some situations to expand the mode shapes to all structural degrees of freedom (dof). This is important as the expanded mode shapes can be used to estimate the damage. For instance, Pandey et al. [1] and Kondo and Hamamoto [2] used the curvature of mode shapes as a damage indicator. An accurate estimation of mode shapes also improves the estimation of stress in structural members for fatigue analysis using vibration data. Pelayo et al. [3] evaluated stresses in a simply supported glass beam and a rectangular glass plate pinned at three points using vibration data and compared them with those estimated using strain gauges attached to some points on the structure. The estimated stresses were found to be in good agreement with those calculated from the strain gauges. A similar study to estimate stresses in an off-shore structure under operational conditions using modal expansion and vibration data was performed by Tarpø et al. [4,5]. Papadimitriou et al. [6] predicted the power spectral densities of stresses in all the locations of a truss by using vibration data obtained at the sensor positions and a dynamic model of the structure. Dertimanis et al. [7] performed a similar study to estimate stresses in a beam due to moving loads. Modal expansion can also be important in industrial structures 
wherein the condition of critical substructures such as tanks and pipelines need to be estimated based on the information provided by sensors attached to the main structure.

Shah and Udwadia [8,9] proposed a methodology for determining the optimal sensor location for identification of dynamic systems under the presence of measurement noise. The optimal configuration was decided as the one which minimizes covariance of the parameter to be estimated. The method was subsequently used to determine the optimal sensor configuration in order to estimate the stiffness of columns of a framed structure using vibration data. Kammer [10] introduced a method which ranks sensor locations based on their contribution to the linear independence of modal displacements. Iteratively, locations that do not contribute significantly are removed. The final sensor configuration tends to maximize trace and determinant of the Fisher information matrix. The method was applied to the selection of sensor locations for identification and correlation of a set of target modes for structural characterization of a large space structure. The effect of both modelling error and measurement noise was further considered in the sensor placement $[11,12]$. Several other criteria exist to measure the suitability of optimal sensor positions such as singular value decomposition [13] and QR decomposition [14] of the modal matrix, the kinetic energy of modes at the sensor positions [15], etc. Kalman filter-based optimal sensor placement methods for state estimation in linear structural systems subjected to unmeasured excitations and noise-contaminated measurements obtained by minimizing the variance of the state estimate are gaining importance [16-18]. In the context of state estimation, mean square error (MSE) based methods are also widely used [19-21]. An excellent overview of previously used optimal sensor placement techniques are available in Dongsheng [22], Ting-Hua and Hong-Nan [23], Mallardo and Aliabadi [24], Gomes et al. [25] and Ostachowicz et al. [26].

In conventional vibration-based monitoring of structures, accelerometers are widely used and one of the commonly used criteria to determine their optimal position involves maximising the linear independence between the modal displacement vectors of different modes reduced to the sensor positions [27]. This is usually achieved by minimizing some scalar metric corresponding to the off-diagonal elements of the Modal Assurance Criterion (MAC) [28] matrix computed at the sensor positions [27,29,30]. However, to the best of authors' knowledge, still, there is no definite proof that such a criterion provides optimal configuration when a modal expansion is needed in the presence of modelling error and measurement noise. Gomes et al. [31] studied a sensor placement criterion which takes mode shape expansion into account for a plate structure. Modal displacements at sensor positions were expanded using splines and subsequently compared with the complete numerical mode shape. The Frobenius norm of the difference between the expanded and numerical mode shape was used as a measure of the quality of expansion. Similarly, Tong et al. [32] studied the sensor placement in case of a slab structure by using the mean square error (MSE) between the numerical mode shapes and the mode shapes expanded from the sensor positions using cubic interpolation schemes. Meo and Zumpano [33] used the $M S E$ between the numerical mode shape and the mode shapes expanded by spline interpolation in case of a bridge structure to study the efficiency of different sensor placement criteria in modal expansion. The effect of measurement noise and modelling errors were ignored in all these studies [31-33]. Murugan Jaya et al. [34] studied the robustness of the conventional optimal configuration for modal expansion in the presence of modelling error and measurement noise in case of a cantilever beam and an industrial milling tower. The similarity between the expanded and the real mode shapes calculated in terms of the diagonal elements of the corresponding MAC matrix was used as a performance criterion. It was observed that with an increase in modelling error and measurement noise, the correlation decreased rapidly, indicating the expanded mode shape to be significantly different from the real mode shape, showing the need for a robust sensor placement scheme.

In this work, a novel performance metric to measure the similarity between real and expanded mode shapes under the presence of modelling error and measurement noise is introduced which is subsequently minimized in order to obtain 
the new optimal sensor locations. The resulting sensor configuration is the best possible choice for modal expansion which is then compared with the conventional optimal configuration. For this new metric, the performance of sequential and genetic algorithm-based search methods, which provides optimal/sub-optimal solutions with very low computational effort, is also evaluated. Furthermore, the efficiency of the new sensor placement criterion is also numerically evaluated using Monte Carlo simulations for some typical modelling error scenarios.

\section{Optimal Sensor Placement for Modal Expansion}

The experimental modal displacements $\boldsymbol{\Psi \in \mathbb { R } ^ { s \times m }}$ evaluated at the $s$ sensor positions for $m$ modes, needs to be expanded to all the $n$ dof in order to obtain the mode shape $\boldsymbol{\Psi} \in \mathbb{R}^{n \times m}$ of the complete structure. For this, any modal expansion technique can be used. The quality of expansion depends on the choice of sensor location and uncertainties in the determination of $\boldsymbol{\psi}$ which can be due to measurement noise in sensors, numerical errors from system identification, etc. In cases where modal expansion is performed using the a priori information from a numerical model, the quality of expansion is also dependent on the errors in the numerical model. Thus, for a given error/uncertainty in the numerical model and modal displacement $\boldsymbol{\psi}$, it is essential to determine the optimal location of sensors which leads to the most efficient modal expansion.

\subsection{Modelling error and measurement noise in mode shape}

A mismatch between the real mode shapes and the ones derived from a numerical model is always found in practice, typically because of incorrect modelling assumptions, unknown system dynamics, inaccurate knowledge of material properties and structural dimensions, and numerical errors arising from different sources (e.g. inadequate mesh size of finite element model, numerical errors in solver, round-off errors, etc.). The resulting net discrepancy between numerical and real mode shapes is represented by $\boldsymbol{\varepsilon}$ and is hereinafter referred at as the modelling error. Thus, numerical mode shapes $\boldsymbol{\Phi} \in \mathbb{R}^{n \times m}$ are assumed to be equal to the real mode shapes $\boldsymbol{\varphi} \in \mathbb{R}^{n \times m}$ corrupted by the modelling error $\boldsymbol{\varepsilon} \in \mathbb{R}^{n \times m}$ as,

$$
\boldsymbol{\Phi}=\boldsymbol{\varphi}+\varepsilon
$$

By using vibration data measured from the real structure using any sensor configuration $\mathbf{S}$, the corresponding experimental modal displacements can be extracted. Due to measurement noise present in the sensors and numerical errors involved in the identification of mode shapes, the calculated modal displacements will also be different from the real values. This difference in the modal displacement at sensor locations is represented by the measurement noise $\boldsymbol{\eta}$.

The experimental mode shapes $\boldsymbol{\psi}$ at the $s$ measurement location extracted using vibration data from the real structure is thus related to the real mode shapes of the structure at sensor locations $\varphi_{\mathbf{s}} \in \mathbb{R}^{s \times m}$ as,

$$
\boldsymbol{\psi}=\boldsymbol{\varphi}_{\mathrm{s}}+\boldsymbol{\eta}
$$

Reynders et al. [35, 36] studied uncertainties in modal displacements when using a Stochastic Subspace Identification (SSI) algorithm on acceleration data collected from a beam. The uncertainty in the mode shapes for any mode was found to be neither constant for all the dof nor were the values at each $d o f$ clearly proportional to the corresponding modal displacement. Similarly, Döhler et al. [37, 38] obtained the confidence intervals for the mode shapes derived from the SSI algorithm in case of a bridge. Results were similar to those obtained in [35]. This confirms that the variation of errors in mode shapes from measurement noise and system identification cannot be easily generalised and represented for the different $d o f$.

Thus, for any mode $l$ of the system, the measurement noise $\eta^{l} \in \mathbb{R}^{s \times 1}$ is assumed to be Gaussian with 0 mean, uncorrelated and with the same standard deviation $\sigma_{\eta}$ at all sensor locations. Even though Tondreau et al., [39] have shown that the errors in the identified mode shapes are correlated at some $d o f$, in the present study this correlation is not considered. Similarly, the modelling error $\varepsilon^{l} \in \mathbb{R}^{n \times 1}$ is also assumed to be Gaussian with 0 mean, uncorrelated and 
with the same standard deviation $\sigma_{\varepsilon}$ at all the $d o f$. Thus, the error (both due to modelling and measurement noise) in the mode shapes can be quantified using $\sigma_{\varepsilon}$ and $\sigma_{\eta}$. As an example, Fig. 1 shows the $95 \%$ uncertainty bound on the real mode shape $\varphi^{l}$ of a cantilever beam for different values of $\sigma_{\varepsilon}$ when the maximum value of the numerical mode shape is normalised to one. The probability density function $(p d f)$ which follows a normal distribution with the standard deviation $\sigma_{\varepsilon}$ is also plotted at the centre of the beam. A higher value of $\sigma_{\varepsilon}$ means higher uncertainty and vice versa.

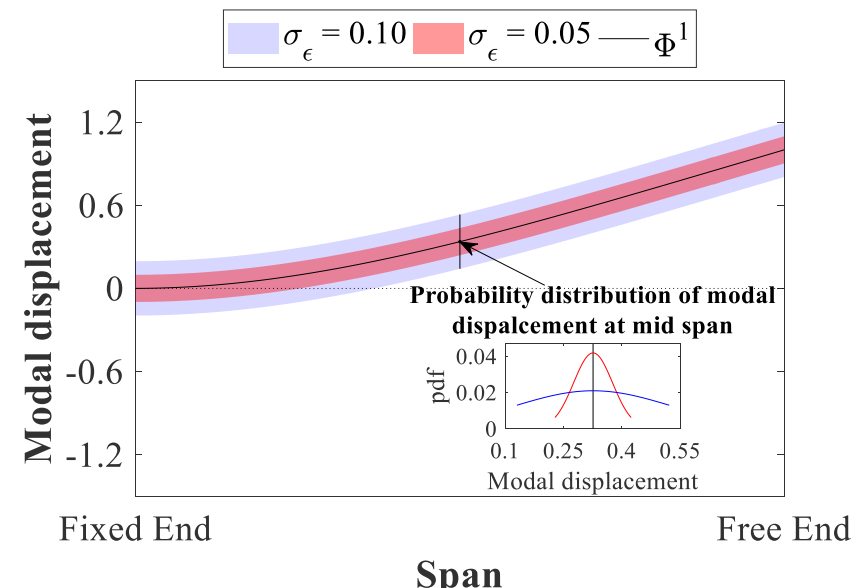

(a)

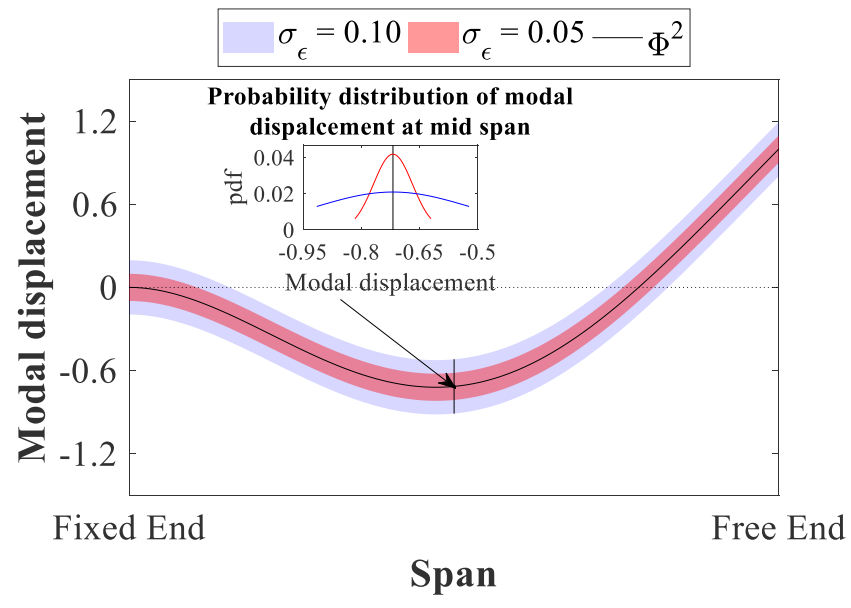

(b)

Fig. 1. 95\% uncertainty bounds in real mode shape $\varphi^{1}$ with standard deviation $\sigma_{\varepsilon}$ of 0.10 and 0.05 in case of a cantilever beam for; (a) Mode-1 and (b) Mode-2

\subsection{Expansion of mode shapes from sparse measurements}

Modal expansion is normally performed in two ways: (a) through a geometric curve fitting using splines or other higherorder polynomial functions without using information from a numerical model or (b) based on the a priori information available from a numerical model. The Guyan static reduction/expansion [40] is one of the first available methods for reduction/expansion of any numerical model. However, since static expansion neglects the inertia of the unmeasured $d o f$, the mode shape predictions can be erroneous if significant masses are located at such dof [41]. This method was extended to include the full equation of motion for modal expansion which resulted in more dynamically accurate methods such as the dynamic expansion method [42]. The present study uses the System Equivalent Reduction Expansion Process (SEREP) [43,44], which expands the mode shapes to unmeasured dof using the complete numerical mode shapes. When the number of sensors $s$ is greater than or equal to the number of modes $m$ used for expansion, $\Psi$ can be expanded to $\boldsymbol{\Psi}$ defined at all the $n$ dof as,

$$
\Psi=\mathbf{C} \boldsymbol{\Psi}
$$

where $\mathbf{C} \in \mathbb{R}^{n x s}$ is the SEREP transformation matrix given by,

$$
\mathbf{C}=\boldsymbol{\Phi} \boldsymbol{\Phi}_{\mathbf{s}}^{\dagger}
$$

$\boldsymbol{\Phi}_{\mathbf{s}} \in \mathbb{R}^{s \times m}$ represents the numerical mode shape at the $s$ sensor positions and $\boldsymbol{\Phi}_{\mathbf{s}}{ }^{\dagger}$ represents the Moore-Penrose pseudo-inverse (left-hand inverse) defined as $\boldsymbol{\Phi}_{\mathbf{s}}{ }^{\dagger}=\left(\boldsymbol{\Phi}_{\mathbf{s}}{ }^{\mathrm{T}} \boldsymbol{\Phi}_{\mathbf{s}}\right)^{-1} \boldsymbol{\Phi}_{\mathbf{s}}{ }^{\mathrm{T}}$.

$\mathbf{C}$ is dependent on the sensor configuration $\mathbf{S}$ and the numerical mode shape $\boldsymbol{\Phi}$. In the absence of measurement noise and modelling error $\left(\sigma_{\eta}=0\right.$ and $\left.\sigma_{\varepsilon}=0\right)$, the experimental modal displacement at the sensor positions will be identical to the corresponding numerical mode shape $\left(\boldsymbol{\Psi}=\boldsymbol{\Phi}_{\mathbf{s}}\right)$. In this case, irrespective of the chosen sensor configuration, the expanded mode shape $\boldsymbol{\Psi}=\boldsymbol{\Phi}$ (this can be verified by substituting $\boldsymbol{\Psi}=\boldsymbol{\Phi}_{\mathbf{s}}$ in Eq. (1)). Thus, under this condition $\boldsymbol{\Psi}$, $\boldsymbol{\varphi}$, and $\boldsymbol{\Phi}$ are all identical thereby making the modal expansion insignificant. The sensor placement problem, thus involves, determination of a certain sensor configuration $\boldsymbol{S}$ to expand the reduced experimental modal displacements $\boldsymbol{\Psi}$ using the numerical mode shapes $\boldsymbol{\Phi}$, such that the expanded mode shapes $\boldsymbol{\Psi}$ are as close as possible to the real mode shapes of the structure $\boldsymbol{\varphi}$ for a non-zero modelling error and measurement noise ( $\sigma_{\eta} \neq 0$ and $\sigma_{\varepsilon} \neq 0$ ). 


\subsection{Normal distance as a measure}

In order to ensure that the expanded mode shapes $\boldsymbol{\Psi}$ are close to the real mode shapes of the structure $\boldsymbol{\varphi}$, a quantitative scalar measure of similarity is required. Consider a $n$ dimensional coordinate system with axes being the dof of the structure. For any mode $l$, the real mode shape $\varphi^{l} \in \mathbb{R}^{n \times 1}$ and the corresponding expanded experimental mode shape $\boldsymbol{\Psi}^{l} \in \mathbb{R}^{n \times 1}$ can be represented as two vectors in this space. Figure 2(a) shows such a system for a 2 dof system when $\boldsymbol{\varphi}^{l}$ and $\boldsymbol{\Psi}^{l}$ are distinct and Fig. 2(b) depicts them being identical but with different scaling. The similarity between these vectors can be quantified measuring either the angle or the distance between them. The $l^{\text {th }}$ diagonal element of MAC matrix calculated between $\boldsymbol{\varphi}$ and $\boldsymbol{\Psi}$ denote the angle, while distance can be measured either in terms of the Euclidean or the normal distance. If the error between the vectors is given by $\mathbf{e}=\boldsymbol{\varphi}^{\boldsymbol{l}}-\boldsymbol{\Psi}^{\boldsymbol{l}}$, the Euclidean distance $\left\|\boldsymbol{\varphi}^{\boldsymbol{l}}-\boldsymbol{\Psi}^{l}\right\|$ is proportional to the square root of $M S E^{l}$ between them and sensor placement based on this criterion was previously used by Papadimitriou et al. [19], Zhang et al. [20] and Soman et al. [21] to estimate stresses, strains, displacements, etc. $M S E^{l}$ is dependent on the scaling of vectors $\Psi^{l}$ and $\varphi^{l}$ and since mode shapes are independent of scaling, in order to use $M S E^{l}$ it should be ensured that both these vectors are scaled identically. Upon scaling the vectors, a closed form expression for $M S E^{l}$ cannot be established easily unless some strong mathematical assumptions are made, which may eventually limit the applicability. It follows from Fig. 2(b) that even when $\boldsymbol{\Psi}^{\boldsymbol{l}}$ and $\boldsymbol{\varphi}^{\boldsymbol{l}}$ are identical but with different scaling, $M S E^{l}$ is not 0 while the normal distance $\left\|\varphi^{l}-\lambda_{c} \boldsymbol{\Psi}^{l}\right\|$ reduces to 0 . The main advantage of using the normal distance $\left\|\boldsymbol{\varphi}^{l}-\lambda_{c} \boldsymbol{\Psi}^{l}\right\|$ is that it allows for obtaining a closed form solution under the assumed measurement noise and modelling errors. Thus, the optimal sensor configuration is the one which minimizes the normal distance between the two vectors. Since both the vectors are defined stochastically, expected value of the normal distance is used for optimization. At a later stage, in order to assess the efficiency of the obtained optimal sensor locations, $M S E^{l}$ is used in a Monte Carlo framework wherein it is possible to scale the mode shape vectors identically.

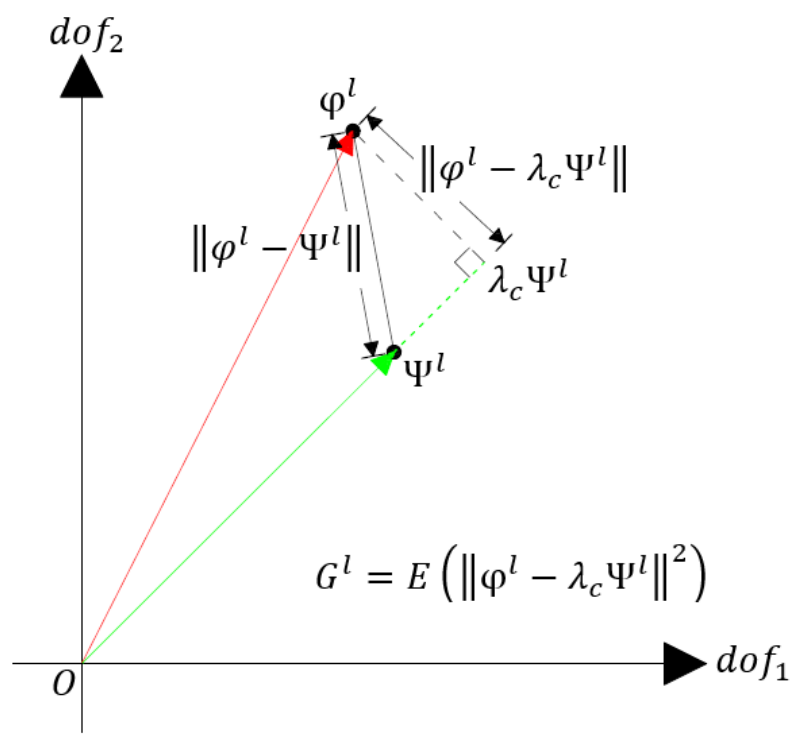

(a)

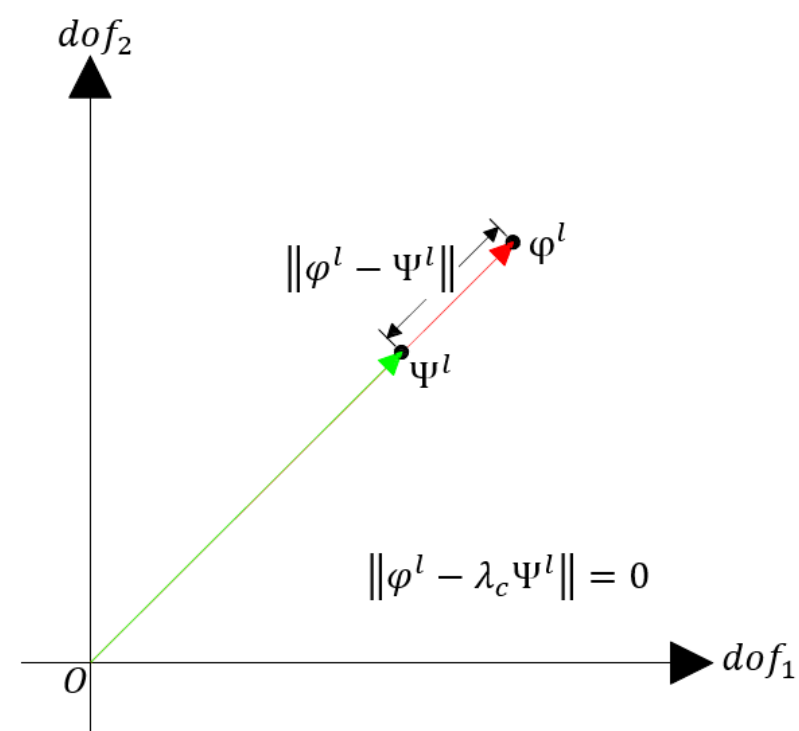

(b)

Fig. 2. Representation of real and expanded experimental mode shape ( $\varphi^{l}$ and $\left.\Psi^{l}\right)$ in a coordinate system with degrees of freedom as axes for a two degree of freedom system when; (a) $\varphi^{l}$ and $\Psi^{l}$ are different and (b) $\varphi^{l}$ and $\Psi^{l}$ are identical but with different scaling

Expected value of the square of the normal distance $G^{l}$ for a particular sensor configuration $\mathbf{S}$ and vectors $\boldsymbol{\varphi}^{l}$ and $\boldsymbol{\Psi}^{l}$ is given by,

$$
G^{l}=\mathrm{E}\left(\boldsymbol{\varphi}^{l^{T}} \boldsymbol{\varphi}^{l}\right)-\frac{\left(\mathrm{E}\left(\boldsymbol{\varphi}^{l^{T}} \boldsymbol{\Psi}^{l}\right)\right)^{2}}{\mathrm{E}\left(\boldsymbol{\Psi}^{l^{T}} \boldsymbol{\Psi}^{l}\right)}
$$


Derivation of $G^{l}$ is shown in Appendix-A. A small value of $G^{l}$ denotes that real mode shape $\varphi^{\mathbf{l}}$ and the expanded mode shape $\boldsymbol{\Psi}^{l}$ are close and vice versa, thereby making it the objective function in this study.

Based on the definition of modelling error and measurement noise in Section 2.1, Eq. (2) becomes,

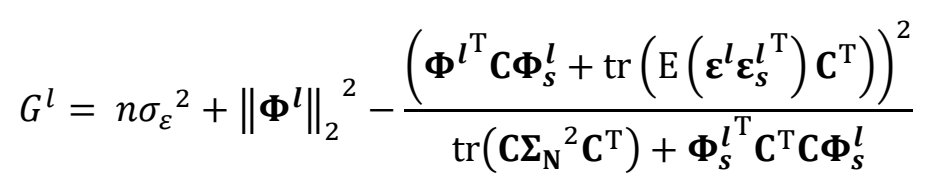

where, $\boldsymbol{\Sigma}_{\mathbf{N}}{ }^{2}=\mathbf{I}_{\mathbf{n}}{\sigma_{\mathrm{N}}}^{2},{\sigma_{\mathrm{N}}}^{2}={\sigma_{\varepsilon}}^{2}+{\sigma_{\eta}}^{2}$ and $\left\|\boldsymbol{\Phi}^{l}\right\|_{2}{ }^{2}=\boldsymbol{\Phi}^{\boldsymbol{l}^{\boldsymbol{T}}} \boldsymbol{\Phi}^{l}$. Appendix-B shows the expected value of individual terms in Eq. (2) which when substituted back results in Eq. (3). Even though the standard deviation of error in modal displacements $\sigma_{\eta}$ due to measurement noise was assumed to be identical for all the sensors, the effect of varying amount of noises across the channels can also be analysed by using

$$
\Sigma_{\mathrm{N}}^{2}=\left[\begin{array}{cccc}
\sigma_{\varepsilon}{ }^{2}+\sigma_{\eta, 1}{ }^{2} & 0 & \cdots & 0 \\
0 & {\sigma_{\varepsilon}{ }^{2}+\sigma_{\eta, 2}{ }^{2}} & \cdots & 0 \\
\vdots & \vdots & \ddots & \vdots \\
0 & 0 & \cdots & \sigma_{\varepsilon}{ }^{2}+\sigma_{\eta, s}{ }^{2}
\end{array}\right]
$$

where $\sigma_{\eta, 1}, \sigma_{\eta, 2}, \ldots, \sigma_{\eta, s}$ are the standard deviations across all the $s$ sensors.

$\mathrm{E}\left(\varepsilon^{l} \varepsilon_{s}^{l^{T}}\right) \in \mathbb{R}^{n \times s}$ is a rectangular covariance matrix between the modelling error at $n$ dof and those at the $s$ sensor positions. As the modelling errors between the different $d o f$ are uncorrelated, elements in the $i^{\text {th }}$ row and $j^{\text {th }}$ column of the matrix is defined as follows,

$$
\mathrm{E}\left(\boldsymbol{\varepsilon}^{l} \boldsymbol{\varepsilon}_{\boldsymbol{s}}^{\boldsymbol{l}^{T}}\right)_{i, j}= \begin{cases}0 & i \neq j^{t h} \text { measured dof } \\ \sigma_{\varepsilon}{ }^{2} & i=j^{t h} \text { measured dof }\end{cases}
$$

By using the definition of $\mathrm{C}$ given in Section 2.2, Eq. (3) can be reduced to the following (Appendix-C shows the derivation),

$$
G^{l}=n \sigma_{\varepsilon}^{2}+\left\|\boldsymbol{\Phi}^{l}\right\|_{2}^{2}-\frac{\left(\left\|\boldsymbol{\Phi}^{l}\right\|_{2}{ }^{2}+{\sigma_{\varepsilon}}^{2} m\right)^{2}}{{\sigma_{\mathrm{N}}}^{2}\left(m+\operatorname{tr}\left(\boldsymbol{\Phi}_{\mathbf{d}}\left(\boldsymbol{\Phi}_{\mathbf{s}}{ }^{\mathrm{T}} \boldsymbol{\Phi}_{\mathbf{s}}\right)^{-1} \boldsymbol{\Phi}_{\mathbf{d}}{ }^{\mathrm{T}}\right)\right)+\left\|\boldsymbol{\Phi}^{l}\right\|_{2}{ }^{2}}
$$

where $\boldsymbol{\Phi}_{\mathbf{d}}$ and $\boldsymbol{\Phi}_{\mathbf{s}}$ represents $\boldsymbol{\Phi}$ partitioned at the unmeasured and measured dof respectively.

It can be seen from Eq. (5) that when modelling error and measurement noise become zero $\left(\sigma_{\varepsilon}=0\right.$ and $\left.\sigma_{\eta}=0\right), G^{l}$ also reduces to zero irrespective of the chosen sensor configuration as explained in Section 2.2. For any given non-zero $\sigma_{\varepsilon}$ and $\sigma_{\eta}$, only the term $\operatorname{tr}\left(\boldsymbol{\Phi}_{\mathbf{d}}\left(\boldsymbol{\Phi}_{\mathbf{s}}{ }^{\mathrm{T}} \boldsymbol{\Phi}_{\mathbf{s}}\right)^{-1} \boldsymbol{\Phi}_{\mathbf{d}}{ }^{\mathrm{T}}\right)$ is dependent on the sensor location. Thus, this term governs the efficiency of each sensor configuration for modal expansion. In case of expanding $m$ modes, the total $G$ over all the modes is calculated as,

$$
G=\sum_{l=1}^{m} G^{l}
$$

\section{Optimization algorithms}

Optimal sensor placement is a combinatorial optimization problem which involves the selection of an optimal set of $s$ sensor positions $\mathbf{S}$ from a set of $r$ possible positions $\mathbf{R}(s<r$ and $\mathbf{S} \subset \mathbf{R})$. Thus, the optimal configuration has to be chosen from a set of $\mathrm{C}_{\mathrm{r}}^{\mathrm{S}}=r ! / s ! /(r-s)$ ! possible number of sensor configurations. In this study, the optimal location is first evaluated using a global search of all the $\mathrm{C}_{\mathrm{r}}^{\mathrm{s}}$ possible sensor configurations. While the formulation of the new metric $G$ is of paramount importance in this work, it is also necessary to understand how $G$ performs when different standard optimization strategies are used. Thus, computationally cheap sequential and meta-heuristic optimization algorithm-based placement methods are introduced as an alternative to the exhaustive search. 


\subsection{Optimal location based on a global search}

In order for the sensor configuration to yield a modal expansion which is as close as possible to the real mode shape, the optimal configuration is calculated by minimizing the function $G$ in Eq. (6). This can be obtained by performing an exhaustive global search of all the $\mathrm{C}_{\mathrm{r}}^{\mathrm{S}}$ possible configurations. Let $\mathbf{S}_{\mathbf{G}}$ denotes the corresponding optimal configuration. The conventional optimal sensor configuration $\mathbf{S}_{\mathbf{c}}$ based on minimizing the linear independence of the modal displacements between the different modes at the sensor locations is also evaluated. This is obtained by minimizing the peak off-diagonal elements of the MAC matrix evaluated for all the modes using the numerical mode shape $\boldsymbol{\Phi}_{s}$. Let $G_{G}$ and $G_{C}$ represent the value of $G$ corresponding to the optimal sensor configuration $\mathbf{S}_{\mathbf{G}}$ and $\mathbf{S}_{\mathbf{c}}$, respectively.

Even though the calculation of the optimal configuration using an exhaustive search of all possible configurations ensures that the resulting solution is the true global optimal, for a large value of possible sensor positions $r$ and number of sensors $s$, the value of $\mathrm{C}_{\mathrm{r}}^{\mathrm{s}}$ become exponentially large. When $r \gg s, \mathrm{C}_{\mathrm{r}}^{\mathrm{s}}$ is of the order of $r^{s} / s$ !. In such cases, instead of performing an exhaustive search, a sequential procedure or any heuristic optimization strategy can be a promising alternative which can provide the optimal or sufficiently accurate sub-optimal results for the function $G$.

\subsection{Sequential sensor placement}

Sequential placement algorithm can be either of a forward or of a backward type depending on whether sensors are added or removed from an initial optimal configuration. The forward sequential placement (FSP) algorithm starts by first placing $m$ (number of modes) sensors in the structure using an exhaustive search by evaluating all the $\mathrm{C}_{\mathrm{r}}^{\mathrm{m}}$ configurations as in Section 3.1 and choosing the configuration $\mathbf{S}_{\text {initial }}$ which minimizes $G$. Now, the remaining $s-m$ sensors are placed in $s-m$ stages to the remaining $r-m$ positions. The $\left(m+1^{\text {th }}\right)$ sensor is placed such that the resulting configuration consisting of $\mathbf{S}_{\mathbf{m + 1}}=\left[\begin{array}{ll}\mathbf{S}_{\text {initial }}{ }^{T} & S_{m+1}\end{array}\right]^{T}$ minimizes $G$. This position $\mathbf{S}_{\mathbf{m + 1}}$ is obtained using an exhaustive search by placing the $m+1^{t h}$ sensor in the remaining $r-m$ positions. Once the $m+1$ sensors are optimally placed, the process is repeated to place the remaining $s-(m+1)$ sensors. The computational effort is now of the order of $r^{m} / m$ ! instead of $r^{s} / s$ ! obtained for an exhaustive global search. Along the same lines, a backward sequential placement (BSP) can also be performed by first keeping sensors at all the $r$ dofs and then successively removing a sensor in each stage by performing an exhaustive search at each of those stages. The BSP algorithm requires the use of only $r(r+1) / 2-s(s+1) / 2$ evaluations. Figure 3 shows a summary of these algorithms.

Both the sequential algorithms are computationally cheap. As an e.g., for placing 6 sensors in 20 possible sensor locations in order to expand 4 modes require $\mathrm{C}_{20}^{6}=38760$ evaluations for an exhaustive search, while the FSP and $\mathrm{BSP}$ requires evaluating only $\mathrm{C}_{20}^{4}+(20-4)+(20-5)=4876$ and $20+19+\cdots+8+7=189$ configurations, respectively. It is to be also noted that the sensor configuration resulting from sequential placement is not guaranteed to be the same as the true global optimal configuration discussed in Section 3.1. However, it was observed that the results from the sequential placement are very close to the global optimal values obtained by the exhaustive search. Let $\mathbf{S}_{\mathbf{F S P}}$ and $\mathbf{S}_{\mathbf{B S P}}$ be the optimal configurations obtained by minimizing $G$ respectively from the FSP and the BSP algorithms and $G_{F S P}$ and $G_{B S P}$ be the corresponding values of $G$. 


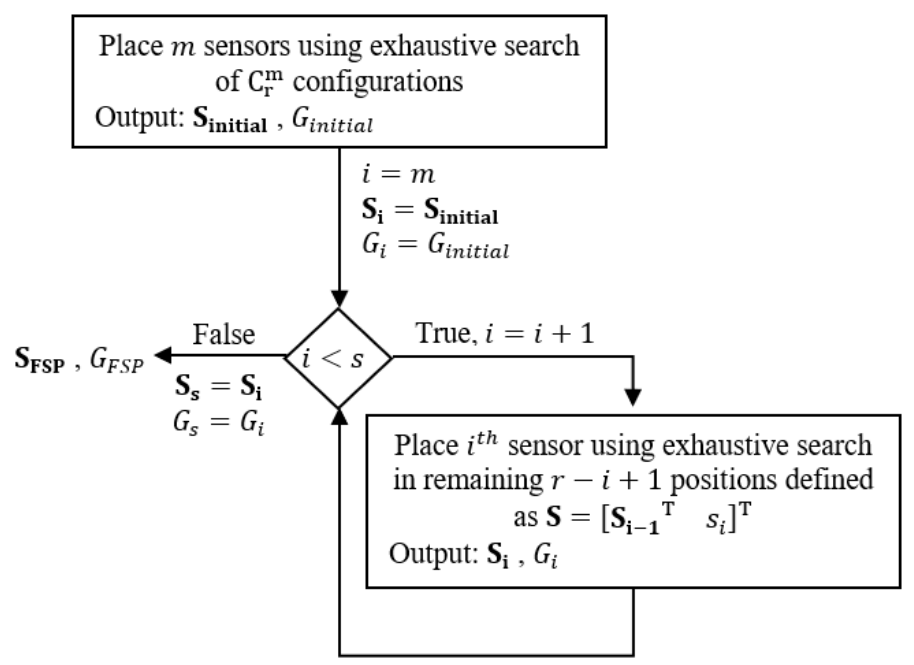

(a)

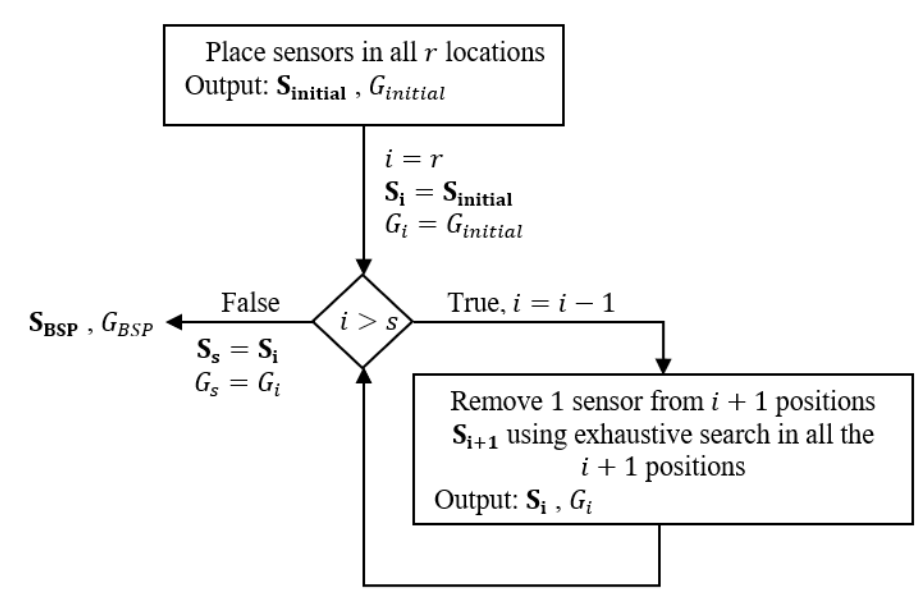

(b)

Fig. 3. Flowchart of sequential sensor placement algorithm; (a) Forward sequential placement and (b) Backward sequential placement.

\subsection{Genetic algorithm-based sensor placement}

In the family of heuristic optimization algorithms used in sensor placement problems, genetic algorithm-based (GA) methods are widely adopted [45-49] and thus is also used in this study. Holland [50, 51] introduced the concept of GA which is a population-based stochastic search technique based on the principles of natural selection and genetics. The method starts by randomly selecting a set of possible initial configurations $\mathbf{X}_{\mathbf{0}}$ which then evolves towards the optimal configuration in each generation. Figure 4 shows a simplified layout of the algorithm. From any generation $i$, the $i+1$ generation is created by means of selection, mutation and crossover [52,53]. Selection involves finding a set of solutions from $\mathbf{X}_{\mathbf{i}}$ which gives the best fitness values. Such solutions are directly included in the next generation $\mathbf{X}_{\mathbf{i}+\mathbf{1}}$. Crossover involves finding new solutions by combining the two best solutions from $\mathbf{X}_{\mathbf{i}}$, while mutation involves generating new solutions by applying random changes to the individual solution in $\mathbf{X}_{\mathbf{i}}$. This process is repeated until some desired convergence criterion is satisfied. In this study, an integer-valued (corresponding to the numbering given to sensor locations) GA is implemented using MATLAB [54]. In order to ensure that the solutions remain integer, special crossover and mutation function given by Deep et al. [55] is used. The fitness function, in this case, is defined as the sum of the objective function $(G)$ to be minimized and a special penalty function for constraint violations [54, 56]. Constraints are provided such that no two sensors takes the same position. Let $\mathbf{S}_{\mathbf{G A}}$ represent the optimal configuration based on this method and $G_{G A}$ denote the corresponding function $G$.

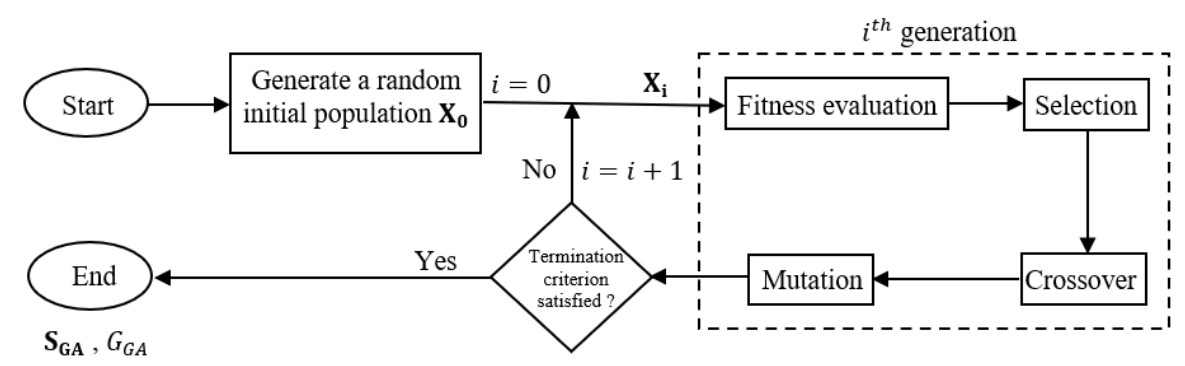

Fig. 4. Flowchart of genetic algorithm-based optimization [49]

\section{Performance Evaluation}

By an exhaustive search of all possible configurations, the new optimal location $\mathbf{S}_{\mathbf{G}}$ obtained by minimizing $G$ is compared with the conventional MAC-based sensor placement $\mathbf{S}_{\mathbf{c}}$. Besides introducing a new metric which can be used as a measure of the accuracy of expansion and thereby determine the new optimal configuration suitable for expansion, 
it is important to understand as to how the conventional optimal configuration $\mathbf{S}_{\mathbf{c}}$ perform in cases where a modal expansion is required. To the best of the authors' knowledge, optimal sensor placement algorithms for modal expansion in the past has not considered the effect of modelling error and measurement noise and were based on the use of mathematical interpolation algorithms (e.g. cubic, spline interpolation, etc.) for expansion. On the contrary, the present sensor placement study considers the effect of both modelling error and measurement noise along with the SEREP modal expansion technique and thus, the new optimal configuration $\mathbf{S}_{\mathbf{G}}$ is not compared with the literature [31-33]. In case of complex structures containing critical substructures, it may be needed to expand the modal displacement of these substructures using the sensor data available only from the main structure. A mathematical interpolation-based expansion may not be preferred in such cases and methods similar to SEREP expansion are needed.

The performances in modal expansion between the different methods are compared first using a simple cantilever model and then with a real industrial milling tower. Smaller the function $G$, better is the similarity between the expanded and the real mode shape. Percentage decrease $I_{C, G}$ in the function $G$, when using the optimal configurations $\mathbf{S}_{\mathbf{G}}$ instead of the conventional optimal configuration $\mathbf{S}_{\mathbf{c}}$, is computed as,

$$
I_{C, G}=\frac{G_{c}-G_{G}}{G_{c}} \times 100
$$

For the function $G$, sequential and GA-based search is also performed. The reduction in $G$ when using the sequential and GA based search is quantified using $I_{C, F S P}, I_{C, B S P}$ and $I_{C, G A}$ and are obtained by replacing $G_{G}$ with $G_{F S P}, G_{B S P}$ and $G_{G A}$ respectively in Eq. (7). A smaller value of $I_{C, G}$ denotes the performance of conventional optimal configuration $\mathbf{S}_{\mathbf{C}}$ in mode shape expansion to be closer to the new optimal configuration $\mathbf{S}_{\mathbf{G}}$ and vice versa. In order to assess the efficiency of the GA and the sequential sensor placement algorithms, the percentage difference in $G_{G}$ with $G_{G A}, G_{F S P}$ and $G_{B S P}$ is evaluated as in Eq. (7) to obtain $I_{G A, G}, I_{F S P, G}$ and $I_{B S P, G}$ by replacing $G_{C}$ with $G_{G A}, G_{F S P}$ and $G_{B S P}$ respectively. Smaller the value of these metrics, better the performance of the corresponding algorithm with respect to the exhaustive global search and vice versa.

The optimal sensor locations based on $G$ are insensitive to the values of $\sigma_{\varepsilon}$ and $\sigma_{\eta}$ (only when $\sigma_{\eta}$ is identical across all the sensors). However, the magnitude of $G$ increases with an increase in $\sigma_{\varepsilon}$ and $\sigma_{\eta}$. $\sigma_{\varepsilon}$ depends on many factors such as knowledge of system dynamics, uncertainties in structure, modelling assumptions, etc. while $\sigma_{\eta}$ depends on the quality of the sensors, cables and data acquisition devices, errors arising in system identification algorithms, etc. [57]. Due to such randomness, the value of $\sigma_{\varepsilon}$ and $\sigma_{\eta}$ is highly problem dependent and thus cannot be generalized. This is also clear from the uncertainty bounds estimated in the SSI of modal parameters for a bridge and a building reported in Reynders et al. [35] where the obtained uncertainty bounds were found to be different for the two cases. Hence, in this study, the modal expansion performance is evaluated for some range of $\sigma_{\varepsilon}$ and $\sigma_{\eta}$ values shown in Table-1.

It is to be noted that while the magnitude of $G$ is dependent on the modes considered and the values of $\sigma_{\varepsilon}$ and $\sigma_{\eta}$, the optimal configuration $\mathbf{S}_{\mathbf{G}}$ is independent of these factors. This is because the effect of sensor configuration is identical in all the modes and thus finding the optimal configuration for a particular mode ensures that it is also optimal for other modes considered in the formulation of the transformation matrix C. Unless mentioned explicitly, $G$ is calculated with respect to the first mode of the structure $\left(G=G^{1}\right)$.

Table - 1. Standard deviation combinations of measurement noise and modelling error

\begin{tabular}{cccccccccc}
\hline \hline Combination I.D. & 1 & 2 & 3 & 4 & 5 & 6 & 7 & 8 & 9 \\
\hline$\sigma_{\eta}$ & 0.01 & 0.01 & 0.01 & 0.05 & 0.05 & 0.05 & 0.10 & 0.10 & 0.10 \\
$\sigma_{\varepsilon}$ & 0.01 & 0.05 & 0.10 & 0.01 & 0.05 & 0.10 & 0.01 & 0.05 & 0.10 \\
\hline \hline
\end{tabular}




\subsection{Cantilever beam}

A 2D cantilever beam was considered, the numerical model of which was created using 100 2-noded Euler-Bernoulli beam elements. Only the translational dof in $\mathrm{Y}$ direction was considered. The first four predominant modes were considered for expansion and numerical mode shapes were scaled such that the maximum magnitude of displacement in each mode was one. 20 possible locations to place sensors which are uniformly spaced along the span of the beam were considered and are shown in Fig. 5.

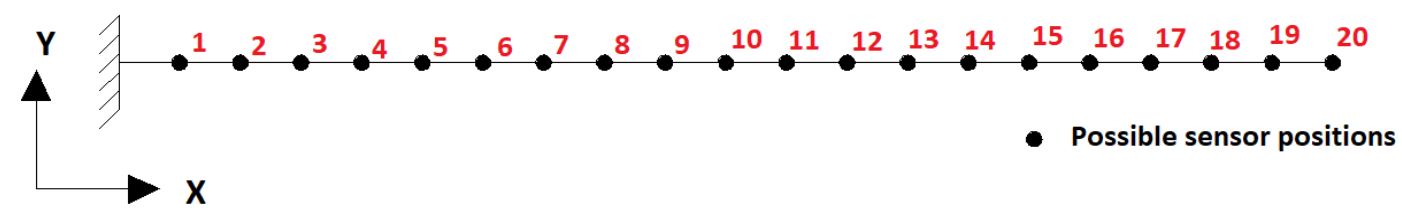

Fig. 5. Cantilever beam showing 20 possible sensor locations $S$ to keep uniaxial accelerometers in $Y$ direction

A simple case of identifying the first 2 predominant modes in $Y$ direction using 2 sensors is initially considered. An exhaustive search of all the $C_{20}^{2}=190$ configurations were carried out to find both the optimal configurations $\mathbf{S}_{\mathbf{C}}$ and $\mathbf{S}_{\mathbf{G}}$. Figure 6 shows the variation of the function $G$ for the first mode and standard deviation combination 9 (from Table1) with the position of both the sensors $s_{1}$ and $s_{2}$.

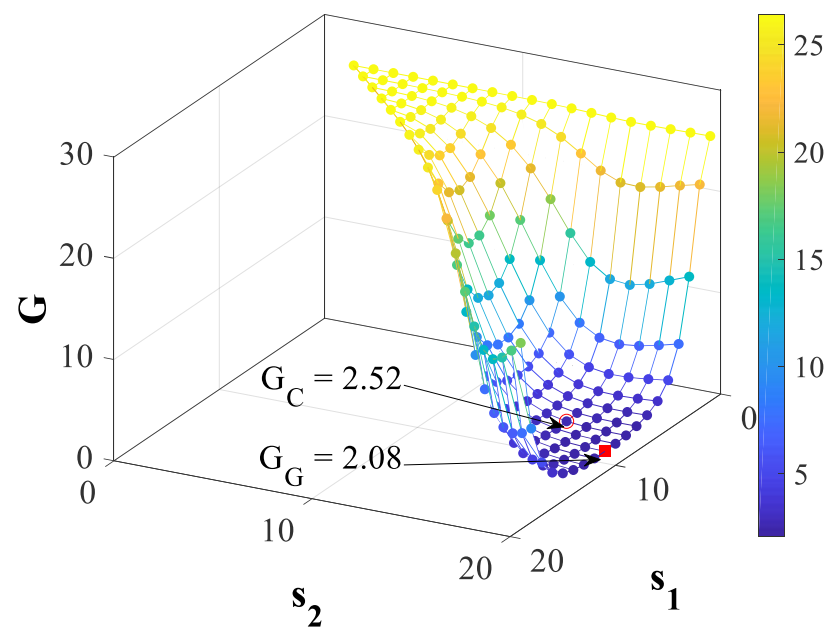

Fig. 6. Variation of $G$ (for mode 1 and standard deviation combination 9) with sensor positions $s_{1}$ and $s_{2}$

It was found that $\mathbf{S}_{\mathbf{C}}=\left[\begin{array}{ll}9 & 17\end{array}\right]^{T}$ and $\mathbf{S}_{\mathbf{G}}=\left[\begin{array}{ll}11 & 20\end{array}\right]^{T}$ and the corresponding $G$ was 2.52 and 2.08, respectively. In this case, $I_{C, G}=17.5 \%$ which denotes a significant reduction in $G$. Thus, an optimization based on $G$ is essential is expected to provide the best sensor configuration for modal expansion.

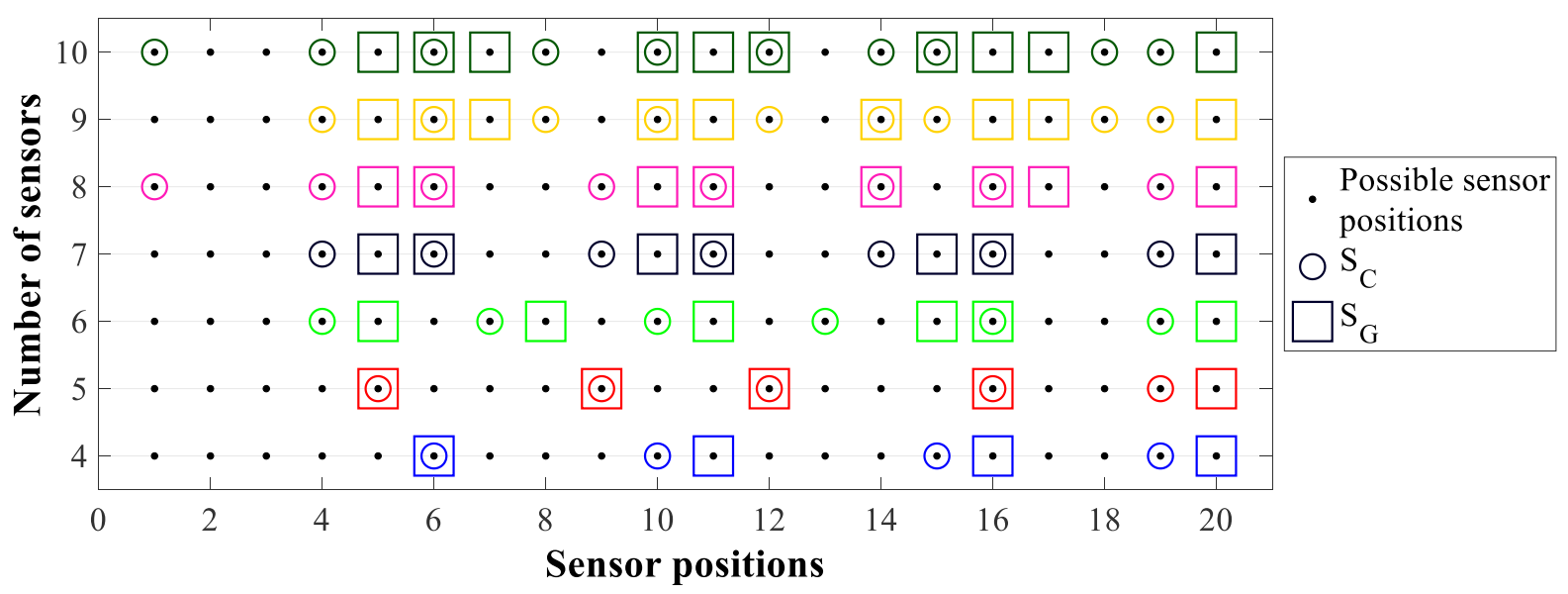

(a) 


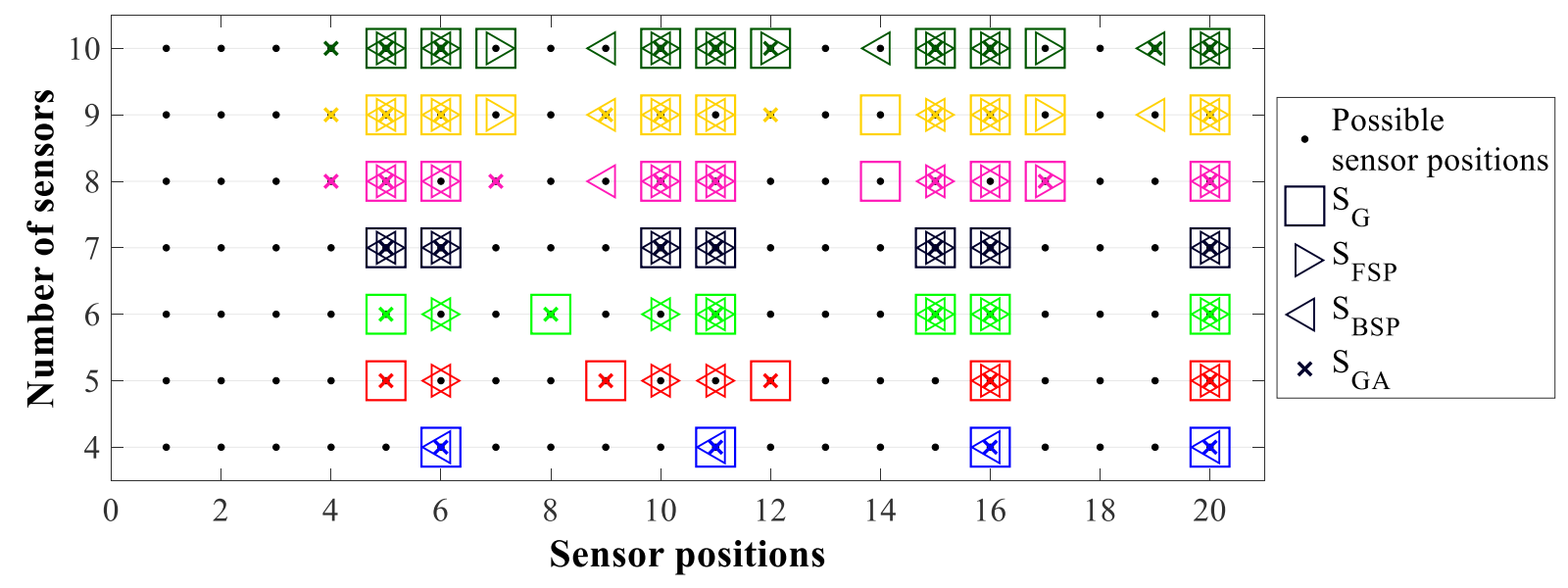

(b)

Fig. 7. Comparison of optimal configurations in case of the cantilever beam for different number of sensors; (a) $S_{\mathrm{C}}$ with $S_{\mathrm{G}}$ and (b) $S_{\mathrm{G}}$ with $S_{\mathrm{FSP}}, S_{\mathrm{BSP}}$ and $S_{\mathrm{GA}}$

Now, a further set of simulation using 4 modes and number of sensors $s$ ranging from 4 to 10 is analysed to obtain the optimal configurations $\mathbf{S}_{\mathbf{C}}$ and $\mathbf{S}_{\mathbf{G}}$, and thus study the influence of the number of sensors on mode shape expansion $G$. Being a theoretical study, the upper limit on the number of sensors was chosen such that they are half the possible number of sensor positions. For cases where $s$ is between 5 to 10, FSP algorithm was used to obtain the optimal configuration $\boldsymbol{S}_{\mathrm{FSP}}$. BSP and GA was also used respectively in order to determine the optimal configurations $\mathbf{S}_{\mathrm{BSP}}$ and $\mathbf{S}_{\mathbf{G A}}$ for $s$ between 4 to 10. In case of GA, population size was taken as 50 and 200 for the case where the number of sensors $s$ is 4 and 10 respectively, while for the remaining cases, it was appropriately chosen between 50 and $200.5 \%$ of the best solutions from any generation was taken directly to the next. The remaining population was created using $80 \%$ of cross-over and $20 \%$ of mutation. The optimization was assumed to converge when the average change in the fitness value over stall generations became less than a tolerance value (1E - 6) [54]. All the GA results shown in this study are based on these settings. However, it is possible to further fine-tune the accuracy and computational cost of GA by suitably adjusting these parameters $[47-49,58]$ and is not attempted here as it is beyond the scope of this paper. Figure 7(a) shows the comparison between $\mathbf{S}_{\mathbf{C}}$ and $\mathbf{S}_{\mathbf{G}}$ while Fig. 7(b) compares $\mathbf{S}_{\mathbf{G}}$ with $\mathbf{S}_{\mathbf{F S P}}, \mathbf{S}_{\mathbf{B S P}}$, and $\mathbf{S}_{\mathbf{G A}}$.

Figure 8 shows the variation of $G_{\mathrm{C}}$ and $G_{\mathrm{G}}$ for $s$ varying from 4 to 10 . It shows that with an increase in $\sigma_{\varepsilon}$ and $\sigma_{\eta}$, both $G_{\mathrm{C}}$ and $G_{\mathrm{G}}$ increases. For any given $\sigma_{\varepsilon}$ and $\sigma_{\eta}, G_{\mathrm{C}}$ and $G_{\mathrm{G}}$ decreases with an increase in the number of sensors $s$. As expected, $G_{\mathrm{G}}$ is always less than $G_{\mathrm{C}}$. Figure 9 (a) shows the variation of $I_{\mathrm{C}, \mathrm{G}}$ for all standard deviation combinations and it is found to vary between 3 to $24 \%$ making the configuration $\mathbf{S}_{\mathbf{G}}$ the best choice for expansion. However, neither the configuration $\mathbf{S}_{\mathbf{G}}$ nor increasing the number of sensors help in offsetting the effect of high $\sigma_{\varepsilon}$ and $\sigma_{\eta}$. Given these limitations, the configuration $\mathbf{S}_{\mathbf{G}}$ is guaranteed to outperform the conventional optimal configuration $\mathbf{S}_{\mathbf{C}}$.

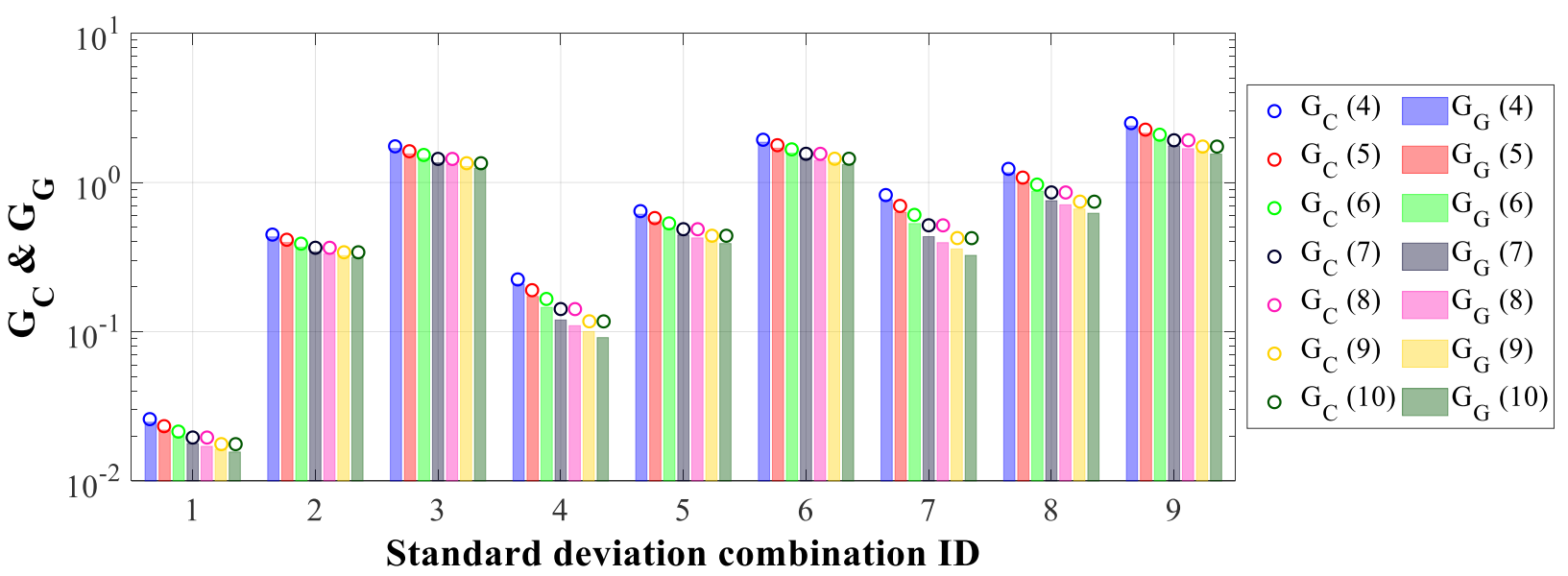


Fig. 8. Comparison of $G_{C}$ and $G_{G}$ in the case of the cantilever beam for all standard deviation combinations (number in parenthesis of $G_{\mathrm{C}}$ and $G_{\mathrm{G}}$ denote the number of sensors $s$ )

Figure 9(a) also shows the variation of $I_{\mathrm{C}, \mathrm{FSP}}, I_{\mathrm{C}, \mathrm{BSP}}$ and $I_{\mathrm{C}, \mathrm{GA}}$ for all standard deviation combinations. The performance of sequential algorithms and GA can also be seen from Fig. $9(\mathrm{~b}-\mathrm{d})$ wherein the variation of $I_{\mathrm{FSP}, \mathrm{G}}, I_{\mathrm{BSP}, \mathrm{G}}$ and $I_{\mathrm{GA}, \mathrm{G}}$ is plotted for different values of $s$. The maximum value of $I_{\mathrm{FSP}, \mathrm{G}}$ and $I_{\mathrm{BSP}, \mathrm{G}}$ is around $3 \%$ and occurs when $s=5$. This may be because, the difference between configuration $\mathbf{S}_{\mathbf{G}}$ and configurations $\mathbf{S}_{\mathbf{F S P}}$ and $\mathbf{S}_{\mathbf{B S P}}$ (from Fig. 7(b)) is maximum for this case. In the case with 7 and 10 sensors, it is seen that FSP provided the global optimal configuration $\mathbf{S}_{\mathbf{G}}$ while BSP provided the global optimal configuration for 4 and 7 number of sensors. This can be also seen from $I_{\mathrm{FSP}, \mathrm{G}}$ and $I_{\mathrm{BSP}, \mathrm{G}}$ taking 0 for these configurations. For $s$ taking values between 8 and $10, I_{\mathrm{FSP}, \mathrm{G}}$ and $I_{\mathrm{BSP}, \mathrm{G}}$ is less than $1 \%$ for all the standard deviation combinations, indicating that both the sequential algorithms are efficient for a larger number of sensors. The GA method provided the true global optimal solution for $s$ between 4 to 7 . Maximum value of $I_{\mathrm{GA}, \mathrm{G}}$ is found to be around $4 \%$ which was reported when $s=8$. The sequential algorithm provides the global optimal for all values of $s$ only if the optimal sensor configuration $\mathbf{S}_{\mathbf{G}}$ when $s=i-1$, is a subset of the optimal configuration for the case with $s=i$. While this condition cannot be ensured for all structures, it can be seen from Fig. 7(a) that some sensors maintain their positions as the number of sensors are increased because of which the sequential method provided optimal solutions in some cases and good sub-optimal solutions in the remaining situations.

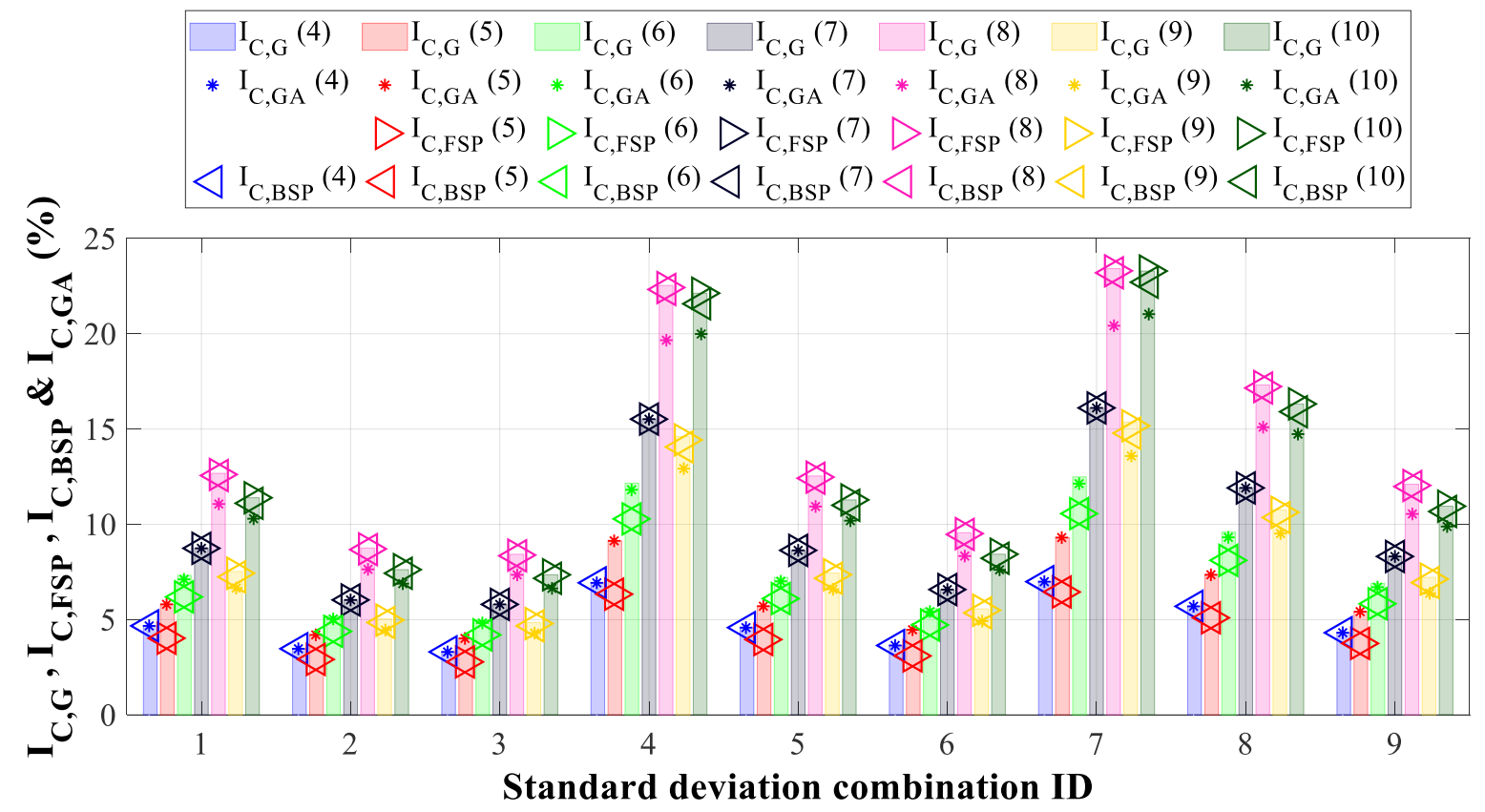

(a)

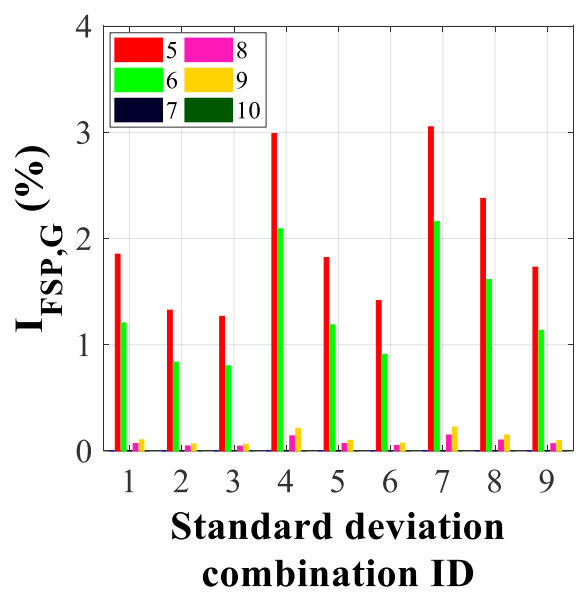

(b)

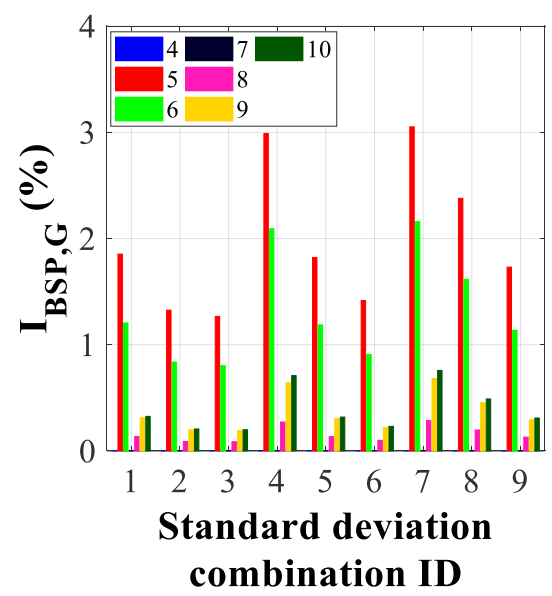

(c)

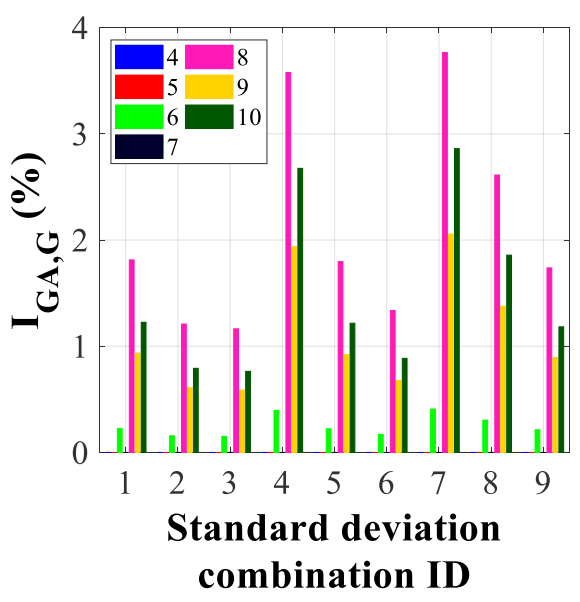

(d) 
Fig. 9. Comparison of different optimization algorithms for the cantilever beam. Variation of (a) $I_{\mathrm{C}, \mathrm{G}}, I_{\mathrm{C}, \mathrm{FSP}}$, $I_{\mathrm{C}, \mathrm{BSP}}$ and $I_{\mathrm{C}, \mathrm{GA}}$, (b) $I_{\mathrm{FSP}, \mathrm{G}}\left(I_{\mathrm{FSP}, \mathrm{G}}=\mathbf{0}\right.$ for $s=7$ and 10), (c) $I_{\mathrm{BSP}, \mathrm{G}}\left(I_{\mathrm{BSP}, \mathrm{G}}=0\right.$ for $s=4$ and 7), and (d) $I_{\mathrm{GA}, \mathrm{G}}$

$$
\left(I_{\mathrm{GA}, \mathrm{G}}=\mathbf{0} \text { for } s=4 \text { to } 7\right)
$$

\subsection{Industrial Milling Tower}

The performance of the proposed sensor placement strategy is now evaluated for an industrial tower of the Birla Carbon Italy SRL production plant in Trecate, Italy. The structure is made of steel with a floor dimension of $6 \times 6.6 \mathrm{~m}$ and approximately $25 \mathrm{~m}$ tall with 7 storeys. It houses two steel tanks at a height of $20 \mathrm{~m}$ and $10 \mathrm{~m}$ from the base. This main tower is attached to a secondary tower which is $30 \mathrm{~m}$ tall with 10 storeys and a floor dimension of $2.5 \times 4.8 \mathrm{~m}$. An expansion is essential in such a structure, especially if the condition of substructures such as the two tanks or other internal pipelines needs to be estimated using sensors located on the main tower. The first and second bending modes of the structure in both the $\mathrm{X}$ and $\mathrm{Y}$ directions are considered for expansion. Figure 10(a) shows a picture of the whole structure while Fig. 10(b) shows the corresponding finite element (FE) model along with the coordinate system. The FE model was created using 3D Euler-Bernoulli beam elements for all the beams and columns while the tanks were modelled using shell elements. The model has 10876 translational dof in $\mathrm{X}$ and $\mathrm{Y}$ direction which is considered for modal expansion. It was decided to provide an identical number of sensors in both the directions. As shown in Fig. 10(c), 22 possible locations for the placement of uniaxial accelerometers were identified in the main tower based on accessibility and other practical constraints. As in the case of the cantilever beam, the effect of sensor configurations $\mathbf{S}_{\mathbf{C}}$, $\mathbf{S}_{\mathbf{G}}$ and $\mathbf{S}_{\mathbf{S}}$ are studied by using $4,6,8$ and 10 sensors.

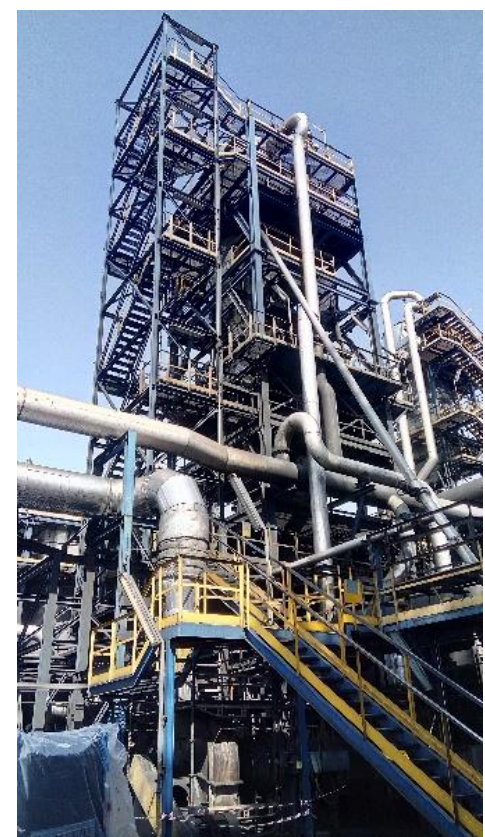

(a)

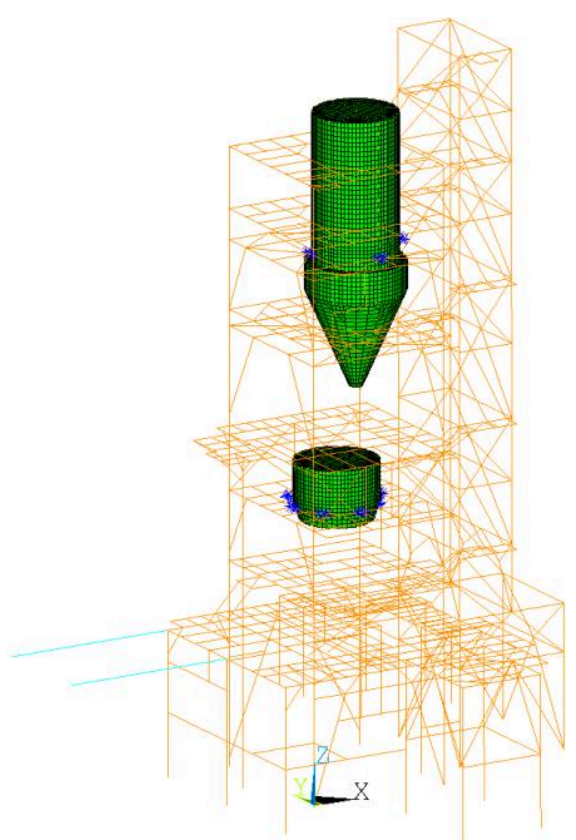

(b)

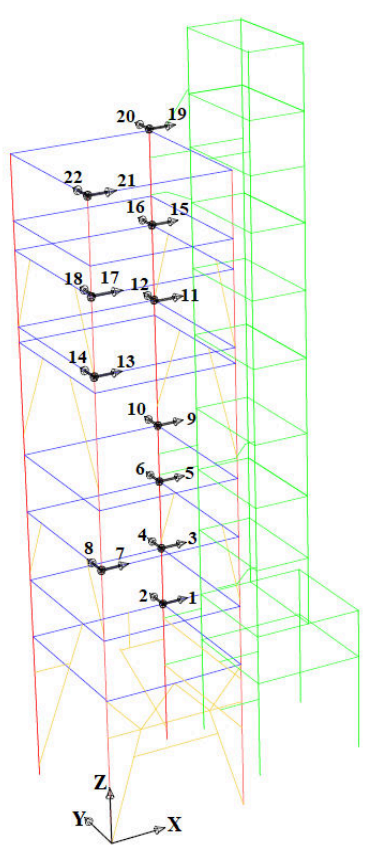

(c)

Fig. 10. (a) Milling tower in Birla Carbon Italy srl, (b) Corresponding finite element model and (c) 22 possible locations for the placement of uniaxial sensors

The mode shapes were normalized such that the maximum displacement in the main tower was one. Figure 11 shows the comparison of the optimal configurations $\mathbf{S}_{\mathrm{C}}, \mathbf{S}_{\mathbf{G}}, \mathbf{S}_{\mathrm{FSP}}, \mathbf{S}_{\mathrm{BSP}}$, and $\mathbf{S}_{\mathrm{GA}}$. GA was implemented with parameters identical to that used for the cantilever beam. Variation of both $G_{\mathrm{C}}$ and $G_{\mathrm{G}}$ for all the standard deviation combinations were similar to that of the cantilever beam and thus is not reported here. 


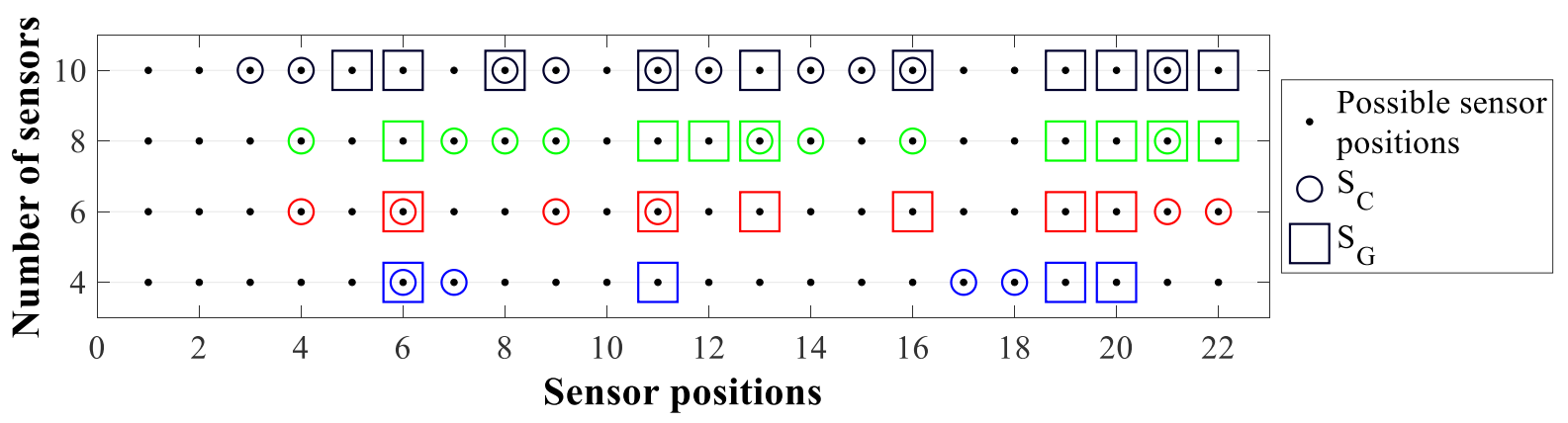

(a)

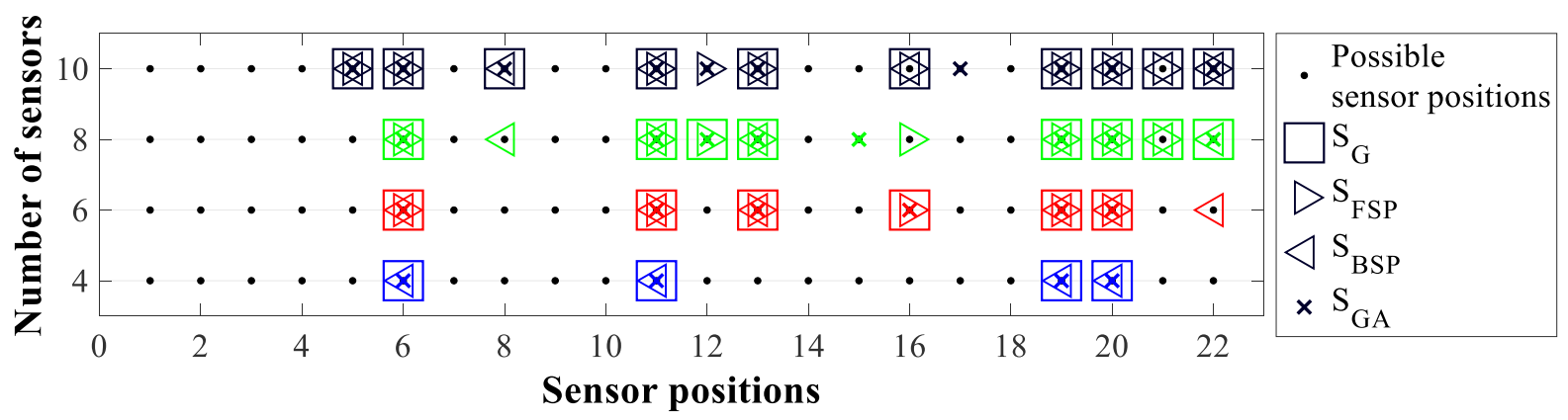

(b)

Fig. 11. Comparison of optimal configurations in case of the milling tower for different number of sensors; (a) $S_{\mathrm{C}}$ with $S_{\mathrm{G}}$ and (b) $S_{\mathrm{G}}$ with $S_{\mathrm{FSP}}, S_{\mathrm{BSP}}$ and $S_{\mathrm{GA}}$

Figure 12(a) shows the function $I_{\mathrm{C}, \mathrm{G}}, I_{\mathrm{C}, \mathrm{FSP}}, I_{\mathrm{C}, \mathrm{BSP}}$, and $I_{\mathrm{C}, \mathrm{GA}}$ for all standard deviation combinations and different values of $s$. It was found that these indices ranged between $10 \%$ to $40 \%$, showing a significant reduction in $G$ when compared to the conventional optimal configuration. Figure 12(b) and 12(c) shows the variation of $I_{\mathrm{FSP}, \mathrm{G}}$ and $I_{\mathrm{BSP}, \mathrm{G}}$, the maximum values of which was only around $2 \%$ and $1 \%$ respectively, while Fig. 12(d) depicts $I_{\mathrm{GA}, \mathrm{G}}$, with a maximum value of $4 \%$ and occured for $s=10$. The FSP method provided the global optimal configuration only in the case with 6 sensors while the BSP method provided global optimal with 4 and 10 sensors. In spite of the fact that both the sequential methods did not result in global optimal for some scenarios, the very low values of $I_{\mathrm{FSP}, \mathrm{G}}$ and $I_{\mathrm{BSP}, \mathrm{G}}$ indicates that they can still be used. GA method provided the optimal configuration in the case with 4 and 6 sensors.

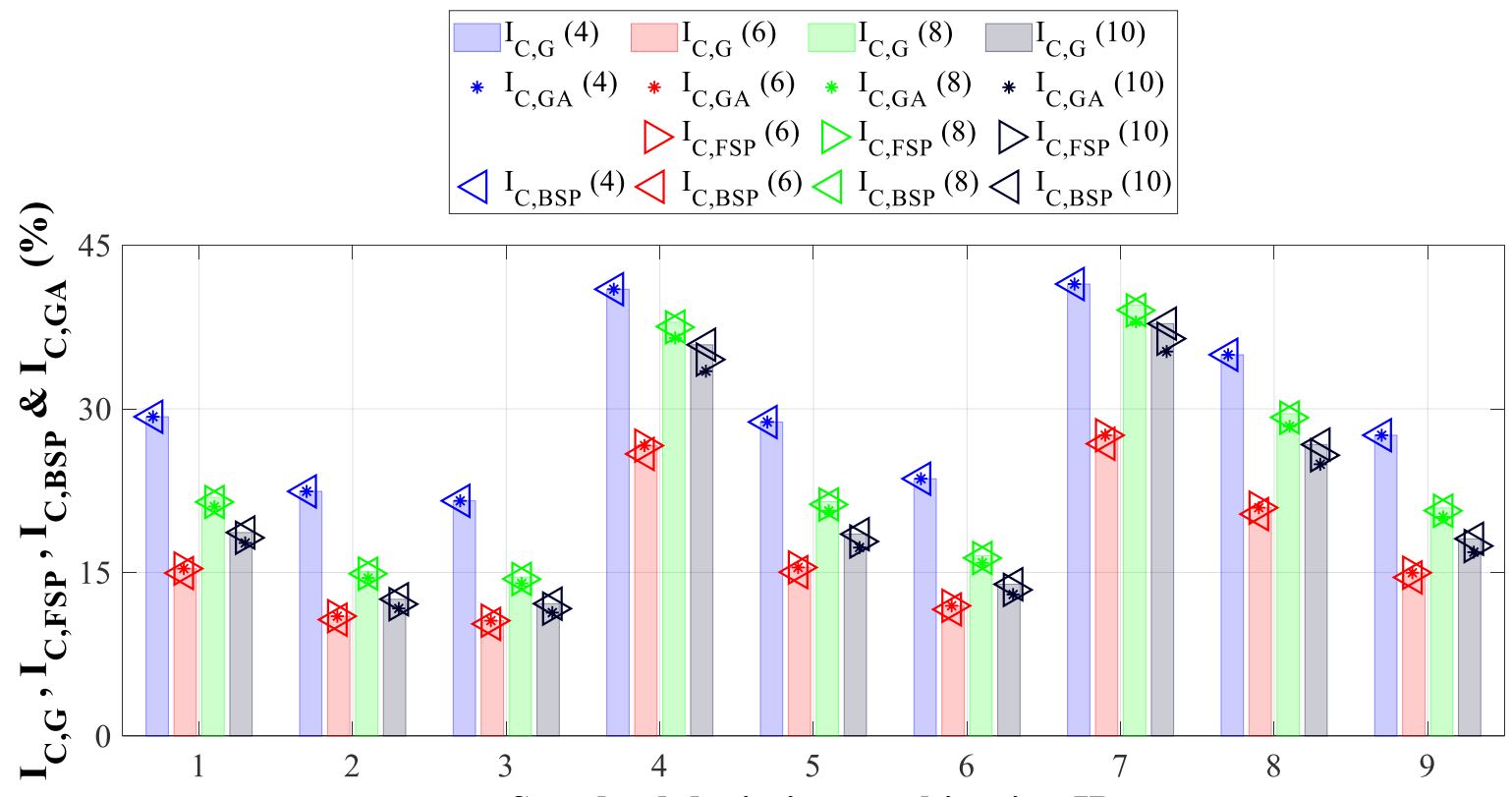

Standard deviation combination ID

(a) 


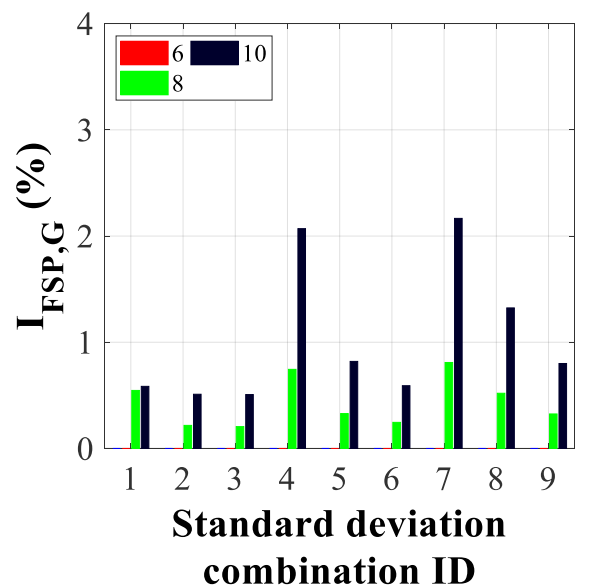

(b)

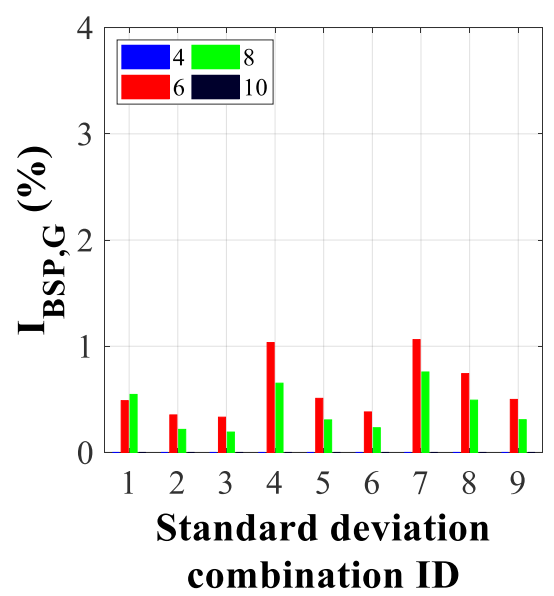

(c)

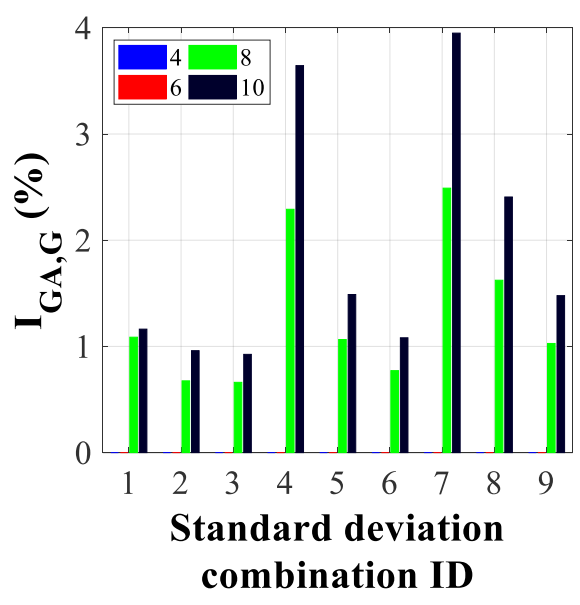

(d)

Fig. 12. Comparison of different algorithms for the milling tower. Variation of (a) $I_{\mathrm{C}, \mathrm{G}}, I_{\mathrm{C}, \mathrm{FSP}}, I_{\mathrm{C}, \mathrm{BSP}}$ and $I_{\mathrm{C}, \mathrm{GA}}$, (b) $I_{\mathrm{FSP}, \mathrm{G}}\left(I_{\mathrm{FSP}, \mathrm{G}}=0\right.$ for $\left.s=6\right)$, (c) $I_{\mathrm{BSP}, \mathrm{G}}\left(I_{\mathrm{BSP}, \mathrm{G}}=0\right.$ for $s=4$ and 10), and (d) $I_{\mathrm{GA}, \mathrm{G}}\left(I_{\mathrm{GA}, \mathrm{G}}=0\right.$ for $s=4$ and 6)

By using the GA based optimization on the function $G$ for both the cantilever beam and the milling tower, it was seen that, the method reached the global optimal solution for cases when the number of sensors are not large, while both the FSP and BSP provided global optimal solution only in certain cases. Still the very low values of $I_{\mathrm{FSP}, \mathrm{G}}$ and $I_{\mathrm{BSP}, \mathrm{G}}$ indicate the closeness of the solutions from the sequential method to the global optimal values. Figure 13 compares the number of configurations evaluated for the cantilever beam and the milling tower when using the different methods. As stated before, the optimization performed using an exhaustive search of all possible configurations becomes expensive as the number of sensors increases. The GA method was found to be computationally cheap for a smaller number of sensors, while with an increase in sensors, it becomes expensive than the sequential methods. As mentioned before, the performance of GA can be further improved by tuning their properties such as the population size, number of elite children, crossovers, and mutations, etc. The BSP is found to be the most computationally efficient method. The FSP is better than GA only for a larger number of sensors. The comparatively better estimation of $G$ coupled with the low computational cost makes the BSP an efficient procedure for optimization in these case studies.

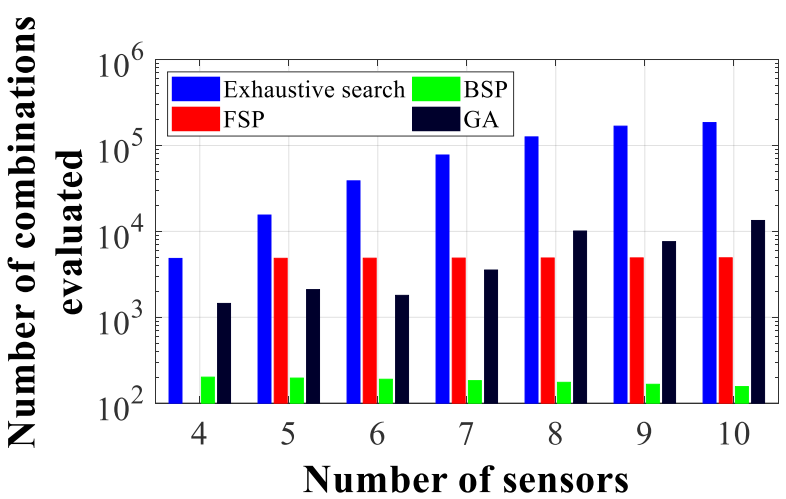

(a)

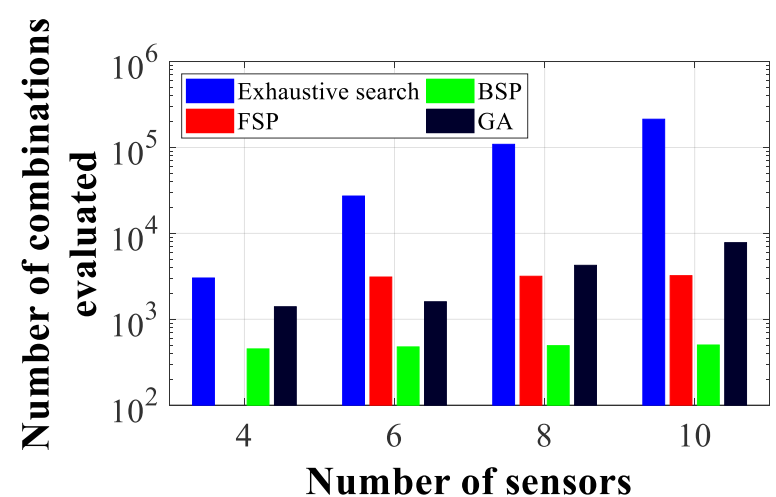

(b)

Fig. 13. Comparison of number of sensor combinations evaluated for different optimization algorithms in case of; (a) The cantilever beam and (b) The milling tower

\section{Performance of the new optimal sensor configuration in modal expansion: A numerical study}

Efficiency in modal expansion using the new optimal sensor locations $\mathrm{S}_{\mathrm{G}}$ over the conventional locations $\mathrm{S}_{\mathrm{C}}$ is evaluated numerically for some typical realistic modelling error scenarios using a Monte Carlo framework [34]. In contrast with the definition of real mode shape $\boldsymbol{\varphi}$ in Section 2.1, here $\boldsymbol{\varphi}$ is obtained directly from the real structural model, which is assumed to be identical to the numerical model with the exception of a known modelling error (bias). The experimental 
mode shape $\boldsymbol{\psi}$ follows the same definition as in Section 2.1 whereby it is assumed to be $\boldsymbol{\varphi}_{\boldsymbol{s}}$ corrupted with a Gaussian measurement noise $\boldsymbol{\eta}$ of zero mean and a given standard deviation $\sigma_{\eta}$ (this is a reasonable assumption, as $\boldsymbol{\eta}$ is not affected by any bias). For a given magnitude of measurement noise, modelling error and number of sensors, the expected value of mean square error $\overline{M S E}$ between $\boldsymbol{\varphi}$ and $\boldsymbol{\Psi}$ evaluated using Monte Carlo simulations is used as a measure of the quality of expansion. Figure 14 shows the algorithm to calculate the same. While the function $G$ was based on the normal distance between the two mode shape vectors, here MSE can be adopted as a metric as both the vectors can be easily normalized. Also, it will be further shown that both $M S E$ and $G$ are analogous. The cantilever beam and the milling tower are again used as case studies. In each case, the configurations $\mathrm{S}_{\mathrm{G}}$ and $\mathrm{S}_{\mathrm{C}}$ are first determined for a given $S$. Subsequently for different values of modelling error in the numerical model and measurement noise, $\overline{M S E}$ corresponding to $\mathrm{S}_{\mathrm{G}}$ and $\mathrm{S}_{\mathrm{C}}$ are evaluated respectively as $\overline{M S E}_{G}$ and $\overline{M S E}_{C}$.

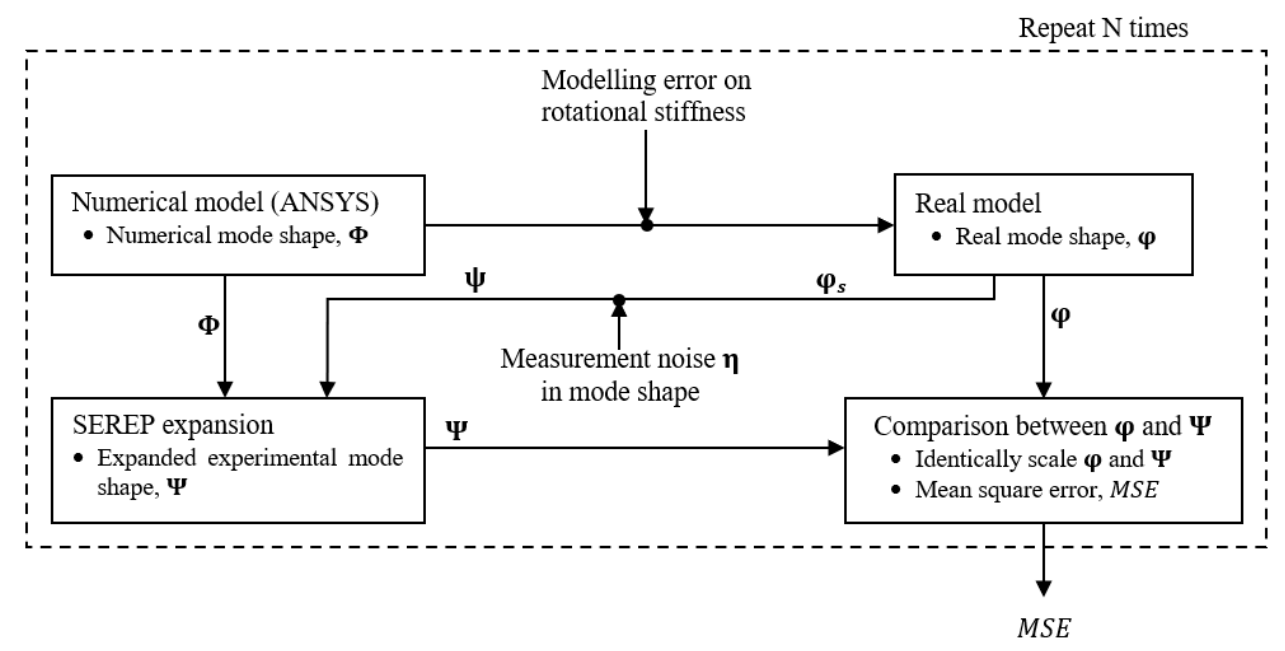

Fig. 14. Flowchart of the Monte Carlo simulations

\subsection{Cantilever beam with modelling error}

A beam identical to that used in Section 4.1 is considered. The rotational stiffness at the fixed end is an important source of uncertainty in such beams (flexible boundary condition). It is assumed that the difference between the numerical and the real structural model is solely attributed to this uncertainty. At the left end of the beam shown in Fig. 5, translational dof in $\mathrm{X}$ and $\mathrm{Y}$ is restrained and a rotational spring $K_{\theta, Z}$ acting along the $\mathrm{Z}$ direction (perpendicular to the plane of the beam) is introduced. The numerical model is formulated by assuming $K_{\theta, Z}=1000 \mathrm{Nm} / \mathrm{rad}$. However, the real value of $K_{\theta, Z}$ is not known and the difference between the numerical and real model is only due to the uncertainty in the values of $K_{\theta, Z}$. In this study, Gaussian distributions with mean $\mu_{K_{\theta, Z}}=1000 \mathrm{Nm} / \mathrm{rad}$ and different values for the coefficient of variation $\operatorname{COV}_{\varepsilon}$ (ratio of the standard deviation to the mean) are used to represent the real values of $K_{\theta, Z}$. As in Section 2.1, measurement noise $\boldsymbol{\eta}$ in mode shape is characterised by a zero-mean Gaussian process with a standard deviation $\sigma_{\eta}$. Based on a sensitivity analysis, the number of simulations $N$ required for Monte Carlo simulations was set to 10000 . In order to identify and expand the first four modes, optimal configurations $\mathbf{S}_{\mathrm{G}}$ and $\mathbf{S}_{\mathrm{C}}$ are calculated for $s=4,6,8$ and 10 using an exhaustive global search and are shown in Table-2.

Table - 2. Optimal sensor location in beam for Monte Carlo study

\begin{tabular}{ccccc}
\hline \hline$s$ & Type & \multicolumn{4}{c}{ Sensor location } \\
\hline \multirow{3}{*}{4} & $\mathbf{S}_{\mathbf{C}}$ & {$\left[\begin{array}{ccccc}4 & 9 & 14 & 18\end{array}\right]^{T}$} \\
& $\mathbf{S}_{\mathbf{G}}$ & {$\left[\begin{array}{cccccc}5 & 10 & 15 & 20\end{array}\right]^{T}$} \\
\hline \multirow{2}{*}{6} & $\mathbf{S}_{\mathbf{C}}$ & {$\left[\begin{array}{llllll}4 & 7 & 10 & 13 & 16 & 19\end{array}\right]^{T}$} \\
& $\mathbf{S}_{\mathbf{G}}$ & {$\left[\begin{array}{llllll}4 & 7 & 10 & 14 & 16 & 20\end{array}\right]^{T}$} \\
\hline
\end{tabular}




\begin{tabular}{|c|c|c|c|c|c|c|c|c|c|c|c|}
\hline \multirow[b]{2}{*}{8} & $\mathbf{S}_{\mathrm{C}}$ & & {$[1$} & 4 & 6 & 9 & 11 & 14 & 16 & $19]^{T}$ & \\
\hline & $\mathbf{S}_{\mathbf{G}}$ & & {$[4$} & 5 & 8 & 10 & 11 & 15 & 16 & $20]^{T}$ & \\
\hline \multirow[b]{2}{*}{10} & $\mathbf{S}_{\mathrm{C}}$ & {$[2$} & 4 & 6 & 8 & 10 & 12 & 14 & 15 & 18 & $19]^{T}$ \\
\hline & $S_{G}$ & {$[4$} & 5 & 6 & 9 & 10 & 11 & 15 & 16 & 17 & $20]^{T}$ \\
\hline
\end{tabular}

Monte Carlo simulations are then performed as in Fig. 14 to obtain the $\overline{M S E}_{G}$ and $\overline{M S E}_{C}$ of the first four modes (scaled for unit modal displacement at the free end) for different values of $s$ and various combinations of $\operatorname{COV}_{\varepsilon}\left(\operatorname{COV}_{\varepsilon}=\right.$ $0.01,0.50,1.00$ and 2.00) and $\sigma_{\eta}\left(\sigma_{\eta}=0.01,0.05,0.10,0.20\right.$ and 0.50$)$. Percentage difference in the $\overline{M S E}$ values when using the new configuration $\mathbf{S}_{\mathbf{G}}$ instead of $\mathbf{S}_{\mathbf{C}}$ is further calculated as $I_{\overline{M S E}}$. Table-3 shows the corresponding values of $\overline{M S E}_{G}, \overline{M S E}_{C}$ and $I_{\overline{M S E}}$. Similar to the variation of $G$ observed in Section 4.1, both $\overline{M S E}_{G}$ and $\overline{M S E}_{C}$ are found to increase with an increase in $\operatorname{COV}_{\varepsilon}$ and $\sigma_{\eta}$ while decreasing with an increase in the number of sensors $s$. For a given $\operatorname{COV}_{\varepsilon}, \sigma_{\eta}$ and $s, \overline{M S E}_{G}$ is significantly lower than $\overline{M S E}_{C}$. This is clear from the values of $I_{\overline{M S E}}$, whose maximum and minimum values are in the order of $42 \%$ and $13.9 \%$ respectively.

Table - 3. $\overline{M S E}_{G}, \overline{M S E}_{C}$ and $I_{\overline{M S E}}$ in case of the beam

\begin{tabular}{|c|c|c|c|c|c|c|c|c|c|c|c|c|c|}
\hline \multirow{3}{*}{$S$} & \multirow{3}{*}{$\sigma_{\eta}$} & \multicolumn{12}{|c|}{$C O V_{\varepsilon}$ of $K_{\theta, Z}$} \\
\hline & & \multicolumn{3}{|c|}{0.01} & \multicolumn{3}{|c|}{0.50} & \multicolumn{3}{|c|}{1.00} & \multicolumn{3}{|c|}{2.00} \\
\hline & & $\overline{M S E}_{G}$ & $\overline{M S E}_{C}$ & $\begin{array}{c}I_{\overline{M S E}} \\
(\%)\end{array}$ & $\overline{M S E}_{G}$ & $\overline{M S E}_{C}$ & $\begin{array}{c}I_{\overline{M S E}} \\
(\%)\end{array}$ & $\overline{M S E}_{G}$ & $\overline{M S E}_{C}$ & $\begin{array}{c}I_{\overline{M S E}} \\
(\%)\end{array}$ & $\overline{M S E}_{G}$ & $\overline{M S E}_{C}$ & $\begin{array}{c}I_{\overline{M S E}} \\
(\%)\end{array}$ \\
\hline \multirow{5}{*}{4} & 0.01 & 0.035 & 0.061 & 42.6 & 0.046 & 0.077 & 40.3 & 0.054 & 0.090 & 40.0 & 0.063 & 0.104 & 39.4 \\
\hline & 0.05 & 0.884 & 1.527 & 42.1 & 0.899 & 1.551 & 42.0 & 0.919 & 1.581 & 41.9 & 0.945 & 1.623 & 41.8 \\
\hline & 0.10 & 3.526 & 5.320 & 33.7 & 3.540 & 5.337 & 33.7 & 3.557 & 5.360 & 33.6 & 3.584 & 5.393 & 33.5 \\
\hline & 0.20 & 11.772 & 14.938 & 21.2 & 11.780 & 14.958 & 21.2 & 11.794 & 14.984 & 21.3 & 11.814 & 15.020 & 21.3 \\
\hline & 0.50 & 34.811 & 42.261 & 17.6 & 34.814 & 42.296 & 17.7 & 34.820 & 42.336 & 17.8 & 34.833 & 42.391 & 17.8 \\
\hline \multirow{5}{*}{6} & 0.01 & 0.026 & 0.032 & 18.8 & 0.035 & 0.044 & 20.5 & 0.042 & 0.053 & 20.8 & 0.050 & 0.063 & 20.6 \\
\hline & 0.05 & 0.642 & 0.811 & 20.8 & 0.657 & 0.829 & 20.7 & 0.675 & 0.852 & 20.8 & 0.698 & 0.881 & 20.8 \\
\hline & 0.10 & 2.584 & 3.200 & 19.3 & 2.598 & 3.215 & 19.2 & 2.616 & 3.236 & 19.2 & 2.643 & 3.265 & 19.1 \\
\hline & 0.20 & 8.893 & 9.933 & 10.5 & 8.903 & 9.944 & 10.5 & 8.917 & 9.959 & 10.5 & 8.939 & 9.982 & 10.4 \\
\hline & 0.50 & 26.309 & 28.692 & 8.3 & 26.321 & 28.704 & 8.3 & 26.336 & 28.720 & 8.3 & 26.361 & 28.746 & 8.3 \\
\hline \multirow{5}{*}{8} & 0.01 & 0.020 & 0.028 & 28.6 & 0.029 & 0.040 & 27.5 & 0.036 & 0.048 & 25.0 & 0.044 & 0.058 & 24.1 \\
\hline & 0.05 & 0.498 & 0.708 & 29.7 & 0.513 & 0.725 & 29.2 & 0.531 & 0.746 & 28.8 & 0.553 & 0.774 & 28.6 \\
\hline & 0.10 & 2.006 & 2.818 & 28.8 & 2.021 & 2.834 & 28.7 & 2.040 & 2.854 & 28.5 & 2.069 & 2.885 & 28.3 \\
\hline & 0.20 & 7.085 & 8.833 & 19.8 & 7.094 & 8.845 & 19.8 & 7.108 & 8.860 & 19.8 & 7.130 & 8.883 & 19.7 \\
\hline & 0.50 & 20.794 & 25.257 & 17.7 & 20.804 & 25.271 & 17.7 & 20.818 & 25.286 & 17.7 & 20.839 & 25.313 & 17.7 \\
\hline \multirow{5}{*}{10} & 0.01 & 0.017 & 0.022 & 22.7 & 0.027 & 0.033 & 18.2 & 0.034 & 0.041 & 17.1 & 0.043 & 0.051 & 15.7 \\
\hline & 0.05 & 0.426 & 0.542 & 21.4 & 0.442 & 0.561 & 21.2 & 0.460 & 0.582 & 21.0 & 0.482 & 0.608 & 20.7 \\
\hline & 0.10 & 1.714 & 2.177 & 21.3 & 1.730 & 2.197 & 21.3 & 1.751 & 2.222 & 21.2 & 1.782 & 2.257 & 21.0 \\
\hline & 0.20 & 6.139 & 7.228 & 15.1 & 6.148 & 7.242 & 15.1 & 6.163 & 7.260 & 15.1 & 6.185 & 7.286 & 15.1 \\
\hline & 0.50 & 17.929 & 20.790 & 13.8 & 17.934 & 20.811 & 13.8 & 17.946 & 20.834 & 13.9 & 17.965 & 20.867 & 13.9 \\
\hline
\end{tabular}

Contrary to the assumptions of identical modelling error $\sigma_{\varepsilon}$ on the real mode shape $\boldsymbol{\varphi}$ at all the dof in Section 2.1 (Fig. 1), it was found from the Monte Carlo simulations that the uncertainty bounds on $\boldsymbol{\varphi}$ with respect to the numerical mode shape $\boldsymbol{\Phi}$ is not constant across all the $d o f$. This is clear from Fig. 15 which shows the uncertainty bounds of $\boldsymbol{\varphi}$ for different values of $\operatorname{COV}_{\varepsilon}$. Even though such significant simplifying assumptions on the effect of modelling error on the real mode shape were made in the formulation of Eq. (5), and thereby the optimal sensor positions $\mathbf{S}_{\mathbf{G}}$, the significantly large values of $I_{\overline{M S E}}$ in Table-3 still demonstrate the usefulness of the new optimal configurations. 


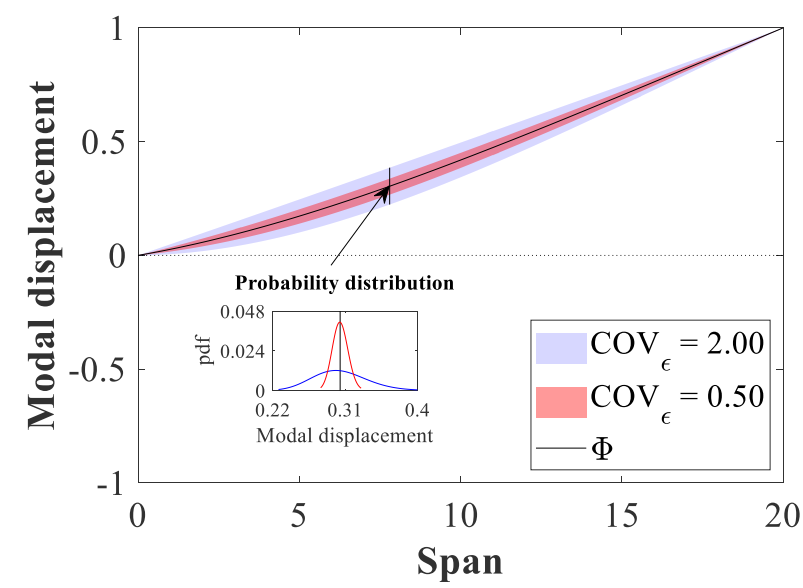

(a)

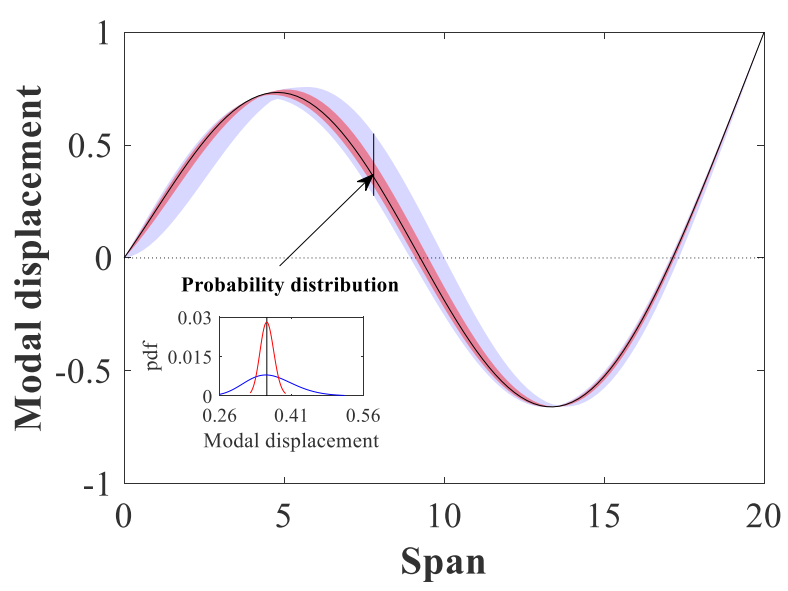

(c)

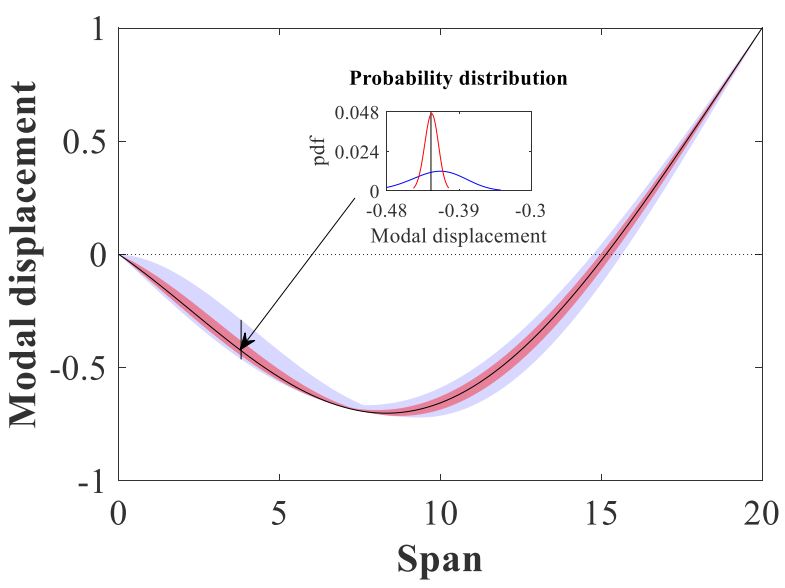

(b)

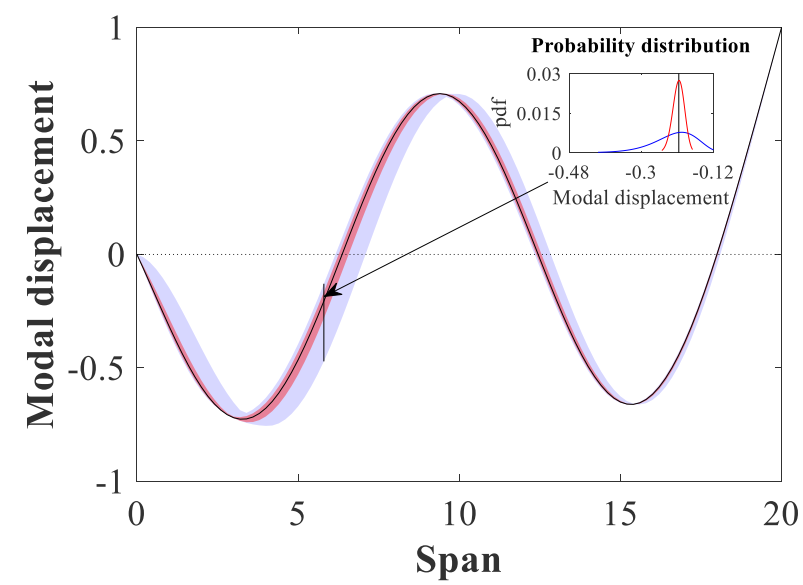

(d)

Fig. 15. 95\% uncertainty bounds on real mode shapes of the cantilever beam from Monte Carlo simulations for; (a) Mode-1, (b) Mode-2, (c) Mode-3 and (d) Mode-4

While the sensor placement criterion in this study was based on $G, \overline{M S E}$ and $G$ are analogous, except that while the former measures the square of Euclidean distance between two vectors, the latter measures the square of the normal distance (which is the shortest distance). As an example, $\overline{\sqrt{M S E^{l}}}$ and $\sqrt{G^{l}}$ for the first four modes of the beam corresponding to some particular values of $\operatorname{COV}_{\varepsilon}, \sigma_{\eta}$ and $s$ are given in Table-4 for both the configurations $\mathbf{S}_{\mathbf{G}}$ and $\mathbf{S}_{\mathbf{C}}$. The variation of both $\overline{\sqrt{M S E^{l}}}$ and $\sqrt{G^{l}}$ is identical. $I \frac{\sqrt{M^{M S}}}{1}$ and $I \sqrt{G^{l}}$, which respectively measures the percentage reduction in $\overline{\sqrt{M S E^{l}}}$ and $\sqrt{G^{l}}$ from the two different sensor configurations, are also calculated. The pattern of $I \frac{\overline{\sqrt{M S E^{l}}}}{}$ and $I_{\sqrt{G l}}$ is found identical, with a difference only in their magnitudes. This indicates that the sensor configurations obtained by minimizing $G$ and that from minimizing $\overline{M S E}$ should be similar.

Table - 4. $\overline{\sqrt{M S E^{l}}}$ and $\sqrt{G^{l}}$ in case of cantilever beam for the first four modes when; (a) $\operatorname{COV}_{\varepsilon}=0.01$,

$$
\sigma_{\eta}=0.01, s=4 \text { and (b) } C O V_{\varepsilon}=2.00, \sigma_{\eta}=0.50, s=10 \text {. }
$$

(a)

\begin{tabular}{ccccccc}
\hline \hline $\begin{array}{c}\text { Mode } \\
\text { Number, } l\end{array}$ & $\sqrt{M S E_{G}^{l}}$ & $\sqrt{M S E_{C}^{l}}$ & $\sqrt{G_{G}^{l}}$ & $\sqrt{G_{C}^{l}}$ & $I \frac{\sqrt{M S E^{l}}}{}(\%)$ & $I_{\sqrt{G^{l}}}(\%)$ \\
\hline 1 & 0.0842 & 0.1093 & 0.0668 & 0.0789 & 23.01 & 15.28 \\
2 & 0.0841 & 0.1042 & 0.0676 & 0.0769 & 19.29 & 12.03 \\
3 & 0.0849 & 0.1020 & 0.0685 & 0.0764 & 16.84 & 10.39 \\
4 & 0.0867 & 0.1045 & 0.0698 & 0.0788 & 17.06 & 11.37 \\
\hline \hline
\end{tabular}


(b)

\begin{tabular}{|c|c|c|c|c|c|c|}
\hline $\begin{array}{c}\text { Mode } \\
\text { Number, } l\end{array}$ & $\overline{\sqrt{M S E_{G}^{l}}}$ & $\sqrt{\sqrt{M S E^{l}{ }_{C}}}$ & $\sqrt{G_{G}^{l}}$ & $\sqrt{G^{l} C}$ & $I \overline{\sqrt{M S E^{l}}}(\%)$ & $I_{\sqrt{G^{l}}}(\%)$ \\
\hline 1 & 1.9825 & 2.1673 & 1.6693 & 1.8662 & 8.53 & 10.55 \\
\hline 2 & 1.9860 & 2.1401 & 1.6786 & 1.8447 & 7.20 & 9.00 \\
\hline 3 & 1.9686 & 2.1293 & 1.6689 & 1.8460 & 7.55 & 9.59 \\
\hline 4 & 1.9946 & 2.0819 & 1.7334 & 1.8289 & 4.19 & 5.22 \\
\hline
\end{tabular}

\subsection{Milling Tower}

In case of the milling tower described in Section 4.2, it is assumed that the rotational stiffness at the connections between the tower and the ground is uncertain. Thus, Monte Carlo simulations similar to those for the cantilever beam are performed by introducing a modelling error in the rotational stiffness at the base of the tower. Translational dof in the nodes of the tower at the base are restrained in all the three directions. Torsional springs of identical stiffness value $K_{\theta}$ are used to model the rotational boundary conditions in all the three axes, the real value of which is unknown. The numerical model is build assuming $K_{\theta}=1 E+06 \mathrm{Nm} / \mathrm{rad}$ while, for the real model, Gaussian distributions with mean $\mu_{K_{\theta}}=1 E+06 \mathrm{Nm} / \mathrm{rad}$ and $C O V_{\varepsilon}$ values of $0.01,0.10,0.50$ and 1.00 is adopted. For $\sigma_{\eta}$, values of 0.01, 0.05, 0.10, 0.50 and 1.00 are used and 4, 6 and 8 number of sensors are considered. The optimal configurations for identifying the first four modes are obtained and are shown in Table-5.

Table - 5. Optimal sensor location in milling tower for Monte Carlo study

\begin{tabular}{|c|c|c|}
\hline$s$ & Type & Sensor location \\
\hline \multirow{2}{*}{4} & $\mathbf{S}_{\mathrm{C}}$ & {$\left[\begin{array}{llll}3 & 6 & 17 & 18\end{array}\right]^{T}$} \\
\hline & $\mathbf{S}_{\mathrm{G}}$ & {$\left[\begin{array}{llll}6 & 11 & 19 & 20\end{array}\right]^{T}$} \\
\hline \multirow[b]{2}{*}{6} & $\mathbf{S}_{\mathrm{C}}$ & {$\left[\begin{array}{llllll}5 & 6 & 10 & 13 & 21 & 22\end{array}\right]^{T}$} \\
\hline & $S_{\mathbf{G}}$ & {$\left[\begin{array}{llllll}6 & 11 & 13 & 16 & 19 & 20\end{array}\right]^{T}$} \\
\hline \multirow[b]{2}{*}{8} & $\mathbf{S}_{\mathrm{C}}$ & {$\left[\begin{array}{llllllll}2 & 4 & 5 & 9 & 11 & 14 & 18 & 19\end{array}\right]^{T}$} \\
\hline & $\mathbf{S}_{\mathrm{G}}$ & {$\left[\begin{array}{llllllll}6 & 8 & 11 & 13 & 19 & 20 & 21 & 22\end{array}\right]^{T}$} \\
\hline
\end{tabular}

Table-6 shows the variation of $\overline{M S E}_{G}, \overline{M S E}_{C}$ and $I_{\overline{M S E}}$ of the first four modes (modal displacement scaled such that the maximum displacement is unity), and it follows the same pattern as in case of the cantilever beam. The maximum and minimum value of $I_{\overline{M S E}}$ are in the order of $60.5 \%$ and $18.4 \%$, which shows a considerable reduction of the $\overline{M S E}$ when using the new configuration $\mathbf{S}_{\mathrm{G}}$ instead of the conventional configuration $\mathbf{S}_{\mathbf{C}}$.

Table - 6. $\overline{M S E}_{G}, \overline{M S E}_{C}$ and $I_{\overline{M S E}}$ in case of milling tower

\begin{tabular}{|c|c|c|c|c|c|c|c|c|c|c|c|c|c|}
\hline \multirow{3}{*}{$S$} & \multirow{3}{*}{$\sigma_{\eta}$} & \multicolumn{12}{|c|}{$C O V_{\varepsilon}$ of $K_{\theta}$} \\
\hline & & \multicolumn{3}{|c|}{0.01} & \multicolumn{3}{|c|}{0.10} & \multicolumn{3}{|c|}{0.50} & \multicolumn{3}{|c|}{1.00} \\
\hline & & $\overline{M S E}_{G}$ & $\overline{M S E}_{C}$ & $\begin{array}{c}I_{\overline{M S E}} \\
(\%)\end{array}$ & $\overline{M S E}_{G}$ & $\overline{M S E}_{C}$ & $\begin{array}{c}I \overline{M S E} \\
(\%)\end{array}$ & $\overline{M S E_{G}}$ & $\overline{M S E}_{C}$ & $\begin{array}{c}I_{\overline{M S E}} \\
(\%)\end{array}$ & $\overline{M S E}_{G}$ & $\overline{M S E}_{C}$ & $\begin{array}{c}I_{\overline{M S E}} \\
(\%)\end{array}$ \\
\hline \multirow{5}{*}{4} & 0.01 & 2.5 & 6.2 & 59.7 & 2.7 & 6.7 & 59.7 & 3.0 & 7.7 & 61.0 & 3.4 & 8.6 & 60.5 \\
\hline & 0.05 & 62.2 & 156.7 & 60.3 & 66.4 & 164.1 & 59.5 & 72.7 & 170.0 & 57.2 & 78.7 & 174.0 & 54.8 \\
\hline & 0.10 & 245.5 & 554.5 & 55.7 & 256.3 & 562.9 & 54.5 & 263.2 & 566.0 & 53.5 & 268.0 & 568.5 & 52.9 \\
\hline & 0.50 & 4287.1 & 6147.4 & 30.3 & 4297.9 & 6149.8 & 30.1 & 4303.0 & 6166.4 & 30.2 & 4306.2 & 6174.5 & 30.3 \\
\hline & 1.00 & 8711.4 & 9408.6 & 7.4 & 8722.1 & 9451.3 & 7.7 & 8728.7 & 9468.9 & 7.8 & 8739.0 & 9484.2 & 7.9 \\
\hline 6 & 0.01 & 1.8 & 2.1 & 14.3 & 2.0 & 2.3 & 13.0 & 2.3 & 2.7 & 14.8 & 2.6 & 3.1 & 16.1 \\
\hline
\end{tabular}




\begin{tabular}{|c|c|c|c|c|c|c|c|c|c|c|c|c|c|}
\hline & 05 & 45.9 & 53.9 & 4.8 & 49.0 & 57.7 & 5.1 & 53.7 & 63.3 & 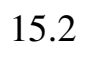 & 58.4 & 69.2 & 15.6 \\
\hline & .10 & 183.0 & 215.1 & 14.9 & 190.7 & 229.0 & 16.7 & 196.0 & 245.6 & 20.2 & 199.5 & 258.3 & 22.8 \\
\hline & 0.50 & 3342.8 & 4008.9 & 16.6 & 3345.1 & 4099.0 & 18.4 & 3352.4 & 4165.9 & 19.5 & 3359.6 & 4201.5 & 20.0 \\
\hline & 1.00 & 7436.7 & 8484.9 & 12.4 & 7438.0 & 8586.2 & 13.4 & 7441.8 & 8632.0 & 13.8 & 7446.1 & 8683.2 & 14.2 \\
\hline \multirow{5}{*}{8} & 0.01 & 1.4 & 1.9 & 26.3 & 1.5 & 2.1 & 28.6 & 1.8 & 2.4 & 25.0 & 2.0 & 2.8 & 28.6 \\
\hline & 0.05 & 34.1 & 47.7 & 28.5 & 36.5 & 50.9 & 28.3 & 40.0 & 56.0 & 28.6 & 43.8 & 61.1 & 28.3 \\
\hline & 0.10 & 136.9 & 190.7 & 28.2 & 143.8 & 199.9 & 28.1 & 151.0 & 207.4 & 27.2 & 156.4 & 212.6 & 26.4 \\
\hline & 0.50 & 2702.2 & 3730.5 & 27.6 & 2721.9 & 3734.8 & 27.1 & 2744.8 & 3750.1 & 26.8 & 2760.4 & 3762.4 & 26.6 \\
\hline & 1.00 & 6747.0 & 8315.5 & 18.9 & 6775.4 & 8342.1 & 18.8 & 6806.7 & 8352.3 & 18.5 & 6827.6 & 8367.7 & 18.4 \\
\hline
\end{tabular}

The Monte-Carlo simulations shows that the optimal configuration $\mathbf{S}_{\mathbf{G}}$ obtained using the novel metric $G$, are robust with respect to both realistic modelling errors and measurement noise in comparison to the conventional configuration $\mathbf{S}_{\mathbf{C}}$. Even when the uncertainty bounds of the real mode shapes vary drastically across the different $d o f$ in contrast to the assumption of identical values for all the $d o f, I \overline{M S E}$ values of the order of $41 \%$ and $60 \%$ were obtained for cantilever beam and milling tower respectively. This indicates that using $\mathbf{S}_{\mathbf{G}}$, a significant improvement in the quality of mode shape expansion is acheived. However, by ensuring maximum linear independence of mode shapes, the conventional configuration $\mathbf{S}_{\mathbf{C}}$ is the best suitable choice for identification problems. Even though the new configuration $\mathbf{S}_{\mathrm{G}}$ provides linearly independent modes, it will not be efficient for identification problems as the conventional configuration $\mathbf{S}_{\mathbf{c}}$. When both mode shape expansion and identification are equally important, a Pareto optimization may be performed.

\section{Conclusions}

Expanding modal displacements from a specific set of sensors to all the degrees of freedom is essential in certain structural health monitoring applications. Most of the commonly used conventional optimal sensor placement strategy aims at maximising the independence of the modal displacements at the sensor positions. However, this is not guaranteed to make the expanded mode shape close to the real mode shape under the presence of modelling error and measurement noise and to date, this specific problem has not been addressed.

In this paper, the normal distance between the expanded and the real mode shape is proposed as a novel tool to quantify their similarity. A new set of optimal configurations were obtained by minimizing this normal distance. The applicability of sequential and genetic algorithm-based techniques for determining the optimal location were also evaluated. It was found that the resulting new configuration, when compared to the conventional configuration, was able to reduce the square of the normal distance by up to $24 \%$ and $40 \%$ respectively in case of the cantilever beam and the milling tower. The efficiency of the obtained sensor locations under some realistic modelling error scenarios were further demonstrated using Monte Carlo simulations. It was also seen that the optimal normal distance increased significantly with an increase in measurement noise and modelling error. However, for a given modelling error and measurement noise, it was possible to improve the quality of modal expansion by increasing the number of sensors. Also, it is to be understood that neither the new optimal configuration nor an increase in the number of sensors is able to balance the increase in the normal distance due to the increase in modelling error and measurement noise. At any rate, for a given modelling error, measurement noise and number of sensors, the new optimal solution will be the best available choice in cases where an expansion is required, ensuring an expansion which is as close as possible to the real mode shape. The numerical Monte Carlo framework for assessing the efficiency of the expansion, may be enhanced by an experimental case study on a benchmark problem in the future. 
Acknowledgment

This study has received funding from the European Union's Horizon 2020 research and innovation programme under the Marie Skłodowska-Curie grant agreement No 721816. The authors would also like to thank Birla Carbon Italy SRL for all the help provided. 


\section{Appendix - A}

\section{A.1. Expected value of normal distance}

Let $\lambda$ represent an arbitrary scaling applied to $\boldsymbol{\Psi}^{\boldsymbol{l}}$. As the Euclidean distance between two distinct vectors is a positive function, minimizing this function is equivalent to minimizing its square. For any sensor configuration $\mathbf{S}$, square of the Euclidean distance $f$ between vectors $\varphi^{l}$ and $\lambda \boldsymbol{\Psi}^{l}$ is given by,

$$
f\left(\boldsymbol{\varphi}^{l}, \lambda \boldsymbol{\Psi}^{l}\right)^{2}=\left\|\boldsymbol{\varphi}^{l}-\lambda \boldsymbol{\Psi}^{l}\right\|^{2}
$$

The expected value of square of the Euclidean distance is given as,

$$
\begin{aligned}
\mathrm{E}\left(f\left(\boldsymbol{\varphi}^{l}, \lambda \boldsymbol{\Psi}^{l}\right)^{2}\right) & =\mathrm{E}\left(\left\|\boldsymbol{\varphi}^{\boldsymbol{l}}-\lambda \boldsymbol{\Psi}^{l}\right\|^{2}\right) \\
& =\mathrm{E}\left(\boldsymbol{\varphi}^{\boldsymbol{l}^{T}} \boldsymbol{\varphi}^{\boldsymbol{l}}\right)-2 \lambda \mathrm{E}\left(\boldsymbol{\varphi}^{\boldsymbol{l}^{T}} \boldsymbol{\Psi}^{l}\right)+\lambda^{2} \mathrm{E}\left(\boldsymbol{\Psi}^{\boldsymbol{l}^{T}} \boldsymbol{\Psi}^{l}\right)
\end{aligned}
$$

Differentiating Eq. (A1.1) with respect to $\lambda$ (for a given $\boldsymbol{\varphi}^{l}$ and $\boldsymbol{\Psi}^{l}$ ) gives,

$$
\begin{aligned}
& \frac{\mathrm{d}}{\mathrm{d} \lambda} \mathrm{E}\left(f\left(\boldsymbol{\varphi}^{l}, \lambda \boldsymbol{\Psi}^{l}\right)^{2}\right)=-2 . \mathrm{E}\left(\boldsymbol{\varphi}^{\boldsymbol{l}^{T}} \boldsymbol{\Psi}^{\boldsymbol{l}}\right)+2 \lambda \mathrm{E}\left(\boldsymbol{\Psi}^{\boldsymbol{l}^{T}} \boldsymbol{\Psi}^{\boldsymbol{l}}\right) \\
& \text { The stationary point of Eq. (A1.1), is } \lambda_{c}=\frac{\mathrm{E}\left(\boldsymbol{\varphi}^{\boldsymbol{l}^{\mathrm{T}}} \boldsymbol{\Psi}^{\boldsymbol{l}}\right)}{\mathrm{E}\left(\boldsymbol{\Psi}^{\boldsymbol{l}^{\mathrm{T}}} \boldsymbol{\Psi}^{\boldsymbol{l}}\right)}
\end{aligned}
$$

The second derivative of Eq. (A1.1) with respect to $\lambda$ is,

$$
\frac{\mathrm{d}^{2}}{\mathrm{~d} \lambda^{2}} \mathrm{E}\left(f\left(\boldsymbol{\varphi}^{l}, \lambda \boldsymbol{\Psi}^{l}\right)^{2}\right)=2 \mathrm{E}\left(\boldsymbol{\Psi}^{\boldsymbol{l}^{T}} \boldsymbol{\Psi}^{l}\right)>0 \forall \boldsymbol{\Psi}^{l} \neq 0
$$

The second derivative is always positive since the expanded mode shape $\boldsymbol{\Psi}^{l}$ can never be a zero vector. Thus, $\lambda_{c}=$ $\mathrm{E}\left(\boldsymbol{\varphi}^{\boldsymbol{l}^{\mathrm{T}}} \boldsymbol{\Psi}^{l}\right) / \mathrm{E}\left(\boldsymbol{\Psi}^{l^{\mathrm{T}}} \boldsymbol{\Psi}^{l}\right)$ corresponds to the minimum of the expected value of the Euclidean norm for mode shapes expanded using a particular sensor configuration $\mathbf{S}$. This happens when the Euclidean norm becomes the normal distance between the two vectors. The square of the expected value of the normal distance $G^{l}$ for a particular sensor configuration S and vectors $\boldsymbol{\varphi}^{l}$ and $\boldsymbol{\Psi}^{l}$ can thus be obtained by substituting $\lambda_{c}$ in Eq. (A.1.1) as,

$$
G^{l}=\mathrm{E}\left(f\left(\boldsymbol{\varphi}^{l}, \lambda_{c} \boldsymbol{\Psi}^{l}\right)^{2}\right)=\mathrm{E}\left(\boldsymbol{\varphi}^{\boldsymbol{l}^{T}} \boldsymbol{\varphi}^{l}\right)-\frac{\left(\mathrm{E}\left(\boldsymbol{\varphi}^{\boldsymbol{l}^{T}} \boldsymbol{\Psi}^{l}\right)\right)^{2}}{\mathrm{E}\left(\boldsymbol{\Psi}^{\boldsymbol{l}^{T}} \boldsymbol{\Psi}^{l}\right)}
$$




\section{Appendix - B}

\section{B.1. Definition of $E\left(\varphi^{l^{T}} \varphi^{l}\right)$}

From the relation between the real mode shape $\varphi^{l}$ and the numerical mode shape $\boldsymbol{\Phi}^{l}$,

$$
\begin{aligned}
& \boldsymbol{\varphi}^{l^{\mathrm{T}}} \boldsymbol{\varphi}^{l}=\left(\Phi^{l}-\varepsilon^{l}\right)^{\mathrm{T}}\left(\Phi^{l}-\varepsilon^{l}\right) \\
& =\Phi^{l^{\mathrm{T}}} \boldsymbol{\Phi}^{l}-\Phi^{l^{\mathrm{T}}} \varepsilon^{l}-\varepsilon^{l^{\mathrm{T}}} \boldsymbol{\Phi}^{l}+\varepsilon^{\boldsymbol{l}^{\mathrm{T}}} \varepsilon^{\boldsymbol{l}} \\
& =\Phi^{l^{\mathrm{T}}} \boldsymbol{\Phi}^{l}-2 \boldsymbol{\Phi}^{l^{\mathrm{T}}} \varepsilon^{l}+\varepsilon^{l^{\mathrm{T}}} \varepsilon^{l} \\
& \mathrm{E}\left(\boldsymbol{\varphi}^{\boldsymbol{l}^{\mathrm{T}}} \boldsymbol{\varphi}^{\boldsymbol{l}}\right)=\mathrm{E}\left(\boldsymbol{\Phi}^{\boldsymbol{l}^{\mathrm{T}}} \boldsymbol{\Phi}^{\boldsymbol{l}}-2 \boldsymbol{\Phi}^{\boldsymbol{l}^{\mathrm{T}}} \boldsymbol{\varepsilon}^{\boldsymbol{l}}+\boldsymbol{\varepsilon}^{\boldsymbol{l}^{\mathrm{T}}} \boldsymbol{\varepsilon}^{\boldsymbol{l}}\right) \\
& =\mathrm{E}\left(\boldsymbol{\Phi}^{l^{\mathrm{T}}} \boldsymbol{\Phi}^{l}\right)-2 \mathrm{E}\left(\boldsymbol{\Phi}^{\boldsymbol{l}^{\mathrm{T}}} \boldsymbol{\varepsilon}^{\boldsymbol{l}}\right)+\mathrm{E}\left(\boldsymbol{\varepsilon}^{\boldsymbol{l}^{\mathrm{T}}} \boldsymbol{\varepsilon}^{\boldsymbol{l}}\right) \\
& =\mathrm{E}\left(\left\|\boldsymbol{\Phi}^{l}\right\|_{2}^{2}\right)-2 \boldsymbol{\Phi}^{l^{\mathrm{T}}} \mathrm{E}\left(\boldsymbol{\varepsilon}^{l}\right)+\mathrm{E}\left(\boldsymbol{\varepsilon}^{\boldsymbol{l}^{\mathrm{T}}} \boldsymbol{\varepsilon}^{\boldsymbol{l}}\right) \\
& =\mathrm{E}\left(\left\|\boldsymbol{\Phi}^{l}\right\|_{2}^{2}\right)-2 \boldsymbol{\Phi}^{l^{\mathrm{T}}} \mathrm{E}\left(\boldsymbol{\varepsilon}^{l}\right)+\mathrm{E}\left(\varepsilon_{1}^{l^{2}}+\varepsilon_{2}^{l^{2}}+\cdots+\varepsilon_{n}^{l^{2}}\right)
\end{aligned}
$$

where, $\boldsymbol{\varepsilon}^{l}=\left[\begin{array}{llll}\varepsilon_{1}^{l} & \varepsilon_{2}^{l} & \cdots & \varepsilon_{n}^{l}\end{array}\right]^{T}$

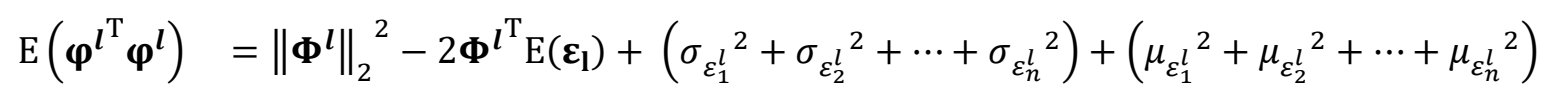

Since, the modelling error is 0 mean with the same standard deviation $\sigma_{\varepsilon}$ at all the $d o f$,

$$
\begin{aligned}
\mathrm{E}\left(\boldsymbol{\varphi}^{l^{\mathrm{T}}} \boldsymbol{\varphi}^{l}\right) & =\left\|\boldsymbol{\Phi}^{l}\right\|_{2}^{2}-0+n \sigma_{\varepsilon}{ }^{2}+0 \\
& =n \sigma_{\varepsilon}{ }^{2}+\left\|\boldsymbol{\Phi}^{l}\right\|_{2}{ }^{2}
\end{aligned}
$$

\section{B.2. Definition of $\mathrm{E}\left(\Psi^{l^{\mathrm{T}}} \Psi^{l}\right)$}

From the definition of experimental modal displacement, $\boldsymbol{\psi}^{l}=\boldsymbol{\varphi}_{s}^{l}+\eta^{l}=\boldsymbol{\Phi}_{s}^{l}-\boldsymbol{\varepsilon}_{s}^{l}+\eta^{l}=\boldsymbol{\Phi}_{s}^{l}-\mathbf{N}^{l}$

where, $\mathbf{N}^{l}=\boldsymbol{\varepsilon}_{\boldsymbol{s}}^{l}-\boldsymbol{\eta}^{l}$ is the net difference of modelling error and measurement noise at the $s$ sensor positions. Since both $\boldsymbol{\varepsilon}$ and $\boldsymbol{\eta}$ are assumed to be 0 mean Gaussian process with the same standard deviations $\sigma_{\varepsilon}$ and $\sigma_{\eta}$ at all the relevant dof, $\mathbf{N}^{l}$ will also be a 0 mean Gaussian process with variance matrix given as,

$$
\boldsymbol{\Sigma}_{\mathbf{N}}{ }^{2}={\sigma_{\mathrm{N}}}^{2} \mathbf{I}_{\mathbf{s}}
$$

where, $\sigma_{\mathrm{N}}^{2}={\sigma_{\varepsilon}}^{2}+{\sigma_{\eta}}^{2}$.

Now,

$$
\begin{aligned}
& \mathrm{E}\left(\boldsymbol{\Psi}^{l}\right)=\boldsymbol{\mu}_{\boldsymbol{\psi}^{l}}=\mathrm{E}\left(\boldsymbol{\Phi}_{s}^{l}-\mathbf{N}^{l}\right)=\boldsymbol{\Phi}_{s}^{l}-\mathrm{E}\left(\mathbf{N}^{l}\right)=\boldsymbol{\Phi}_{s}^{l}
\end{aligned}
$$

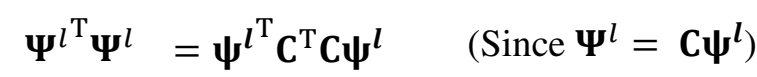

$$
\begin{aligned}
& \mathrm{E}\left(\boldsymbol{\Psi}^{\boldsymbol{l}^{\mathrm{T}}} \boldsymbol{\Psi}^{\boldsymbol{l}}\right)=\mathrm{E}\left(\boldsymbol{\Psi}^{\boldsymbol{l}^{\mathrm{T}}} \mathbf{C}^{\mathrm{T}} \mathbf{C} \boldsymbol{\Psi}^{\boldsymbol{l}}\right) \\
& =\mathrm{E}\left(\left(\boldsymbol{\Psi}^{\boldsymbol{l}}-\boldsymbol{\mu}_{\boldsymbol{\psi}^{l}}\right)^{\mathrm{T}} \mathbf{C}^{\mathrm{T}} \mathbf{C}\left(\boldsymbol{\Psi}^{\boldsymbol{l}}-\boldsymbol{\mu}_{\boldsymbol{\Psi}^{l}}\right)\right)+\boldsymbol{\mu}_{\boldsymbol{\Psi}^{l}} \mathbf{C}^{\mathrm{T}} \mathbf{C} \boldsymbol{\mu}_{\boldsymbol{\Psi}^{l}} \\
& =\mathrm{E}\left(\operatorname{tr}\left(\mathbf{C}\left(\boldsymbol{\Psi}^{l}-\boldsymbol{\mu}_{\boldsymbol{\psi}^{l}}\right)\left(\boldsymbol{\Psi}^{\boldsymbol{l}}-\boldsymbol{\mu}_{\boldsymbol{\psi}^{l}}\right)^{\mathrm{T}} \mathbf{C}^{\mathrm{T}}\right)\right)+\boldsymbol{\mu}_{\boldsymbol{\psi}^{l}}{ }^{\mathrm{T}} \mathbf{C}^{\mathrm{T}} \mathbf{C} \boldsymbol{\mu}_{\boldsymbol{\psi}^{l}} \\
& =\operatorname{tr}\left(\mathbf{C E}\left(\left(\boldsymbol{\Psi}^{l}-\boldsymbol{\mu}_{\boldsymbol{\Psi}^{l}}\right)\left(\boldsymbol{\Psi}^{\boldsymbol{l}}-\boldsymbol{\mu}_{\boldsymbol{\Psi}^{l}}\right)^{\mathrm{T}}\right) \mathbf{C}^{\mathrm{T}}\right)+\boldsymbol{\mu}_{\boldsymbol{\psi}^{l}} \mathbf{C}^{\mathrm{T}} \mathbf{C} \boldsymbol{\mu}_{\boldsymbol{\Psi}^{l}} \\
& =\operatorname{tr}\left(\mathbf{C} \boldsymbol{\Sigma}_{\mathbf{N}}{ }^{2} \mathbf{C}^{\mathrm{T}}\right)+\boldsymbol{\mu}_{\boldsymbol{\psi}^{l}}{ }^{\mathrm{T}} \mathbf{C}^{\mathrm{T}} \mathbf{C} \boldsymbol{\mu}_{\boldsymbol{\psi}^{l}} \text {, where } \boldsymbol{\Sigma}_{\mathbf{N}}{ }^{2} \in \mathbb{R}^{s x s} \text { is the covariance matrix of } \boldsymbol{\Psi}^{\boldsymbol{l}} \text {. } \\
& \mathrm{E}\left(\boldsymbol{\Psi}^{\boldsymbol{l}^{\mathrm{T}}} \boldsymbol{\Psi}^{l}\right)=\operatorname{tr}\left(\mathbf{C} \boldsymbol{\Sigma}_{\mathbf{N}}^{2} \mathbf{C}^{\mathrm{T}}\right)+\boldsymbol{\Phi}_{\boldsymbol{s}}^{\boldsymbol{l}^{\mathrm{T}}} \mathbf{C}^{\mathrm{T}} \mathbf{C} \boldsymbol{\Phi}_{\boldsymbol{s}}^{l}
\end{aligned}
$$


B.2.1. Proof that $E\left(\left(\psi^{l}-\mu_{\psi^{l}}\right)^{T} C^{T} C\left(\psi^{l}-\mu_{\psi^{l}}\right)\right)+\mu_{\psi^{l}}{ }^{T} C^{T} C \mu_{\psi^{l}}=E\left(\psi^{l^{T}} C^{T} C \psi^{l}\right)$

$$
\begin{aligned}
& \mathrm{E}\left(\left(\boldsymbol{\Psi}^{l}-\boldsymbol{\mu}_{\boldsymbol{\psi}^{l}}\right)^{T} \mathbf{C}^{T} \mathbf{C}\left(\boldsymbol{\Psi}^{l}-\boldsymbol{\mu}_{\boldsymbol{\psi}^{l}}\right)\right)+\boldsymbol{\mu}_{\boldsymbol{\psi}^{l}} \mathbf{C}^{\mathrm{T}} \mathbf{C} \boldsymbol{\mu}_{\boldsymbol{\psi}^{l}}
\end{aligned}
$$

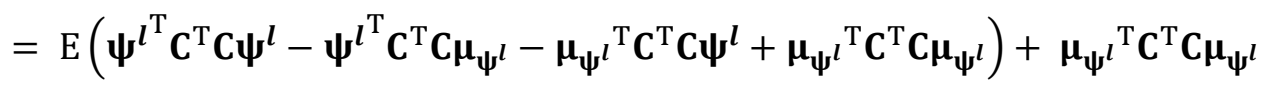

$$
\begin{aligned}
& =\mathrm{E}\left(\boldsymbol{\psi}^{l^{\mathrm{T}}} \mathbf{C}^{\mathrm{T}} \mathbf{C} \boldsymbol{\psi}^{l}\right)-\mathrm{E}\left(\boldsymbol{\psi}^{l^{\mathrm{T}}}\right) \mathbf{C}^{\mathrm{T}} \mathbf{C} \boldsymbol{\mu}_{\psi^{l}}-\boldsymbol{\mu}_{\boldsymbol{\psi}^{l}} \mathbf{C}^{\mathrm{T}} \mathbf{C E}\left(\boldsymbol{\Psi}^{l}\right)+\boldsymbol{\mu}_{\boldsymbol{\psi}^{l}} \mathbf{C}^{\mathrm{T}} \mathbf{C} \boldsymbol{\mu}_{\psi^{l}}+\boldsymbol{\mu}_{\boldsymbol{\psi}^{l}}{ }^{\mathrm{T}} \mathbf{C}^{\mathrm{T}} \mathbf{C} \boldsymbol{\mu}_{\psi^{l}} \\
& =\mathrm{E}\left(\boldsymbol{\psi}^{l^{\mathrm{T}}} \mathbf{C}^{\mathrm{T}} \mathbf{C} \boldsymbol{\psi}^{l}\right)-\boldsymbol{\mu}_{\psi^{l}}{ }^{\mathrm{T}} \mathbf{C}^{\mathrm{T}} \mathbf{C} \boldsymbol{\mu}_{\psi^{l}}-\boldsymbol{\mu}_{\psi^{l}}{ }^{\mathrm{T}} \mathbf{C}^{\mathrm{T}} \mathbf{C} \boldsymbol{\mu}_{\psi^{l}}+\boldsymbol{\mu}_{\psi^{\prime}}{ }^{\mathrm{T}} \mathbf{C}^{\mathrm{T}} \mathbf{C} \boldsymbol{\mu}_{\psi^{l}}+\boldsymbol{\mu}_{\psi^{l}}{ }^{\mathrm{T}} \mathbf{C}^{\mathrm{T}} \mathbf{C} \boldsymbol{\mu}_{\psi^{l}} \\
& =\mathrm{E}\left(\boldsymbol{\psi}^{\boldsymbol{l}^{\mathrm{T}}} \mathbf{C}^{\mathrm{T}} \mathbf{C} \boldsymbol{\psi}^{l}\right)
\end{aligned}
$$

\section{B.3. Definition of $\mathrm{E}\left(\varphi^{l^{\mathrm{T}}} \Psi^{l}\right)$}

$$
\mathrm{E}\left(\boldsymbol{\varphi}^{l^{\mathrm{T}}} \boldsymbol{\Psi}^{l}\right)=\mathrm{E}\left(\boldsymbol{\varphi}^{l^{\mathrm{T}}}\right) \mathrm{E}\left(\boldsymbol{\Psi}^{l}\right)+\operatorname{tr}\left(\operatorname{cov}\left(\boldsymbol{\varphi}^{l}, \boldsymbol{\Psi}^{l}\right)\right)
$$

where $\operatorname{cov}\left(\boldsymbol{\varphi}^{l}, \Psi^{l}\right)$ is the covariance matrix between $\boldsymbol{\varphi}^{l}$ and $\Psi^{l}$

$$
\begin{aligned}
& \mathrm{E}\left(\boldsymbol{\varphi}^{\boldsymbol{l}^{\mathrm{T}}} \boldsymbol{\Psi}^{l}\right)=\mathrm{E}\left(\boldsymbol{\varphi}^{l^{\mathrm{T}}}\right) \mathrm{E}\left(\mathbf{C} \boldsymbol{\Psi}^{l}\right)+\operatorname{tr}\left(\operatorname{cov}\left(\boldsymbol{\varphi}^{l}, \mathbf{C} \boldsymbol{\psi}^{l}\right)\right) \\
& =\boldsymbol{\Phi}^{l^{\mathrm{T}}} \mathbf{C} \boldsymbol{\Phi}_{s}^{l}+\operatorname{tr}\left(\operatorname{cov}\left(\boldsymbol{\varphi}^{l}, \mathbf{C} \boldsymbol{\Psi}^{l}\right)\right) \quad\left(\text { Since } \mathrm{E}\left(\boldsymbol{\varphi}^{l}\right)=\boldsymbol{\Phi}^{l} \text { and } \mathrm{E}\left(\boldsymbol{\Psi}^{l}\right)=\boldsymbol{\Phi}_{s}^{l}\right) \\
& \operatorname{tr}\left(\operatorname{cov}\left(\boldsymbol{\varphi}^{l}, \mathbf{C} \boldsymbol{\Psi}^{l}\right)\right)=\operatorname{tr}\left(\mathrm{E}\left(\left(\boldsymbol{\varphi}^{l}-\boldsymbol{\mu}_{\varphi^{l}}\right)\left(\mathbf{C} \boldsymbol{\Psi}^{l}-\mathbf{C} \boldsymbol{\mu}_{\boldsymbol{\psi}^{l}}\right)^{\mathrm{T}}\right)\right) \\
& =\operatorname{tr}\left(\mathrm{E}\left(\left(\boldsymbol{\Phi}^{l}-\boldsymbol{\varepsilon}^{l}-\boldsymbol{\Phi}^{l}\right)\left(\boldsymbol{\Phi}_{\boldsymbol{s}}^{l}-\boldsymbol{\varepsilon}_{\boldsymbol{s}}^{l}+\boldsymbol{\eta}^{l}-\boldsymbol{\Phi}_{s}^{l}\right)^{\mathrm{T}} \mathbf{C}^{\mathrm{T}}\right)\right) \\
& =\operatorname{tr}\left(\mathrm{E}\left(\left(-\boldsymbol{\varepsilon}^{l}\right)\left(-\boldsymbol{\varepsilon}_{\boldsymbol{s}}^{l}+\boldsymbol{\eta}^{l}\right)^{\mathrm{T}} \mathbf{C}^{\mathrm{T}}\right)\right) \\
& =\operatorname{tr}\left(\mathrm{E}\left(\boldsymbol{\varepsilon}^{l}\left(\boldsymbol{\varepsilon}_{\boldsymbol{s}}^{l}-\boldsymbol{\eta}^{l}\right)^{\mathrm{T}}\right) \mathbf{C}^{\mathrm{T}}\right) \\
& =\operatorname{tr}\left(\mathrm{E}\left(\boldsymbol{\varepsilon}^{l} \boldsymbol{\varepsilon}_{\boldsymbol{s}}^{\boldsymbol{l}^{\mathrm{T}}}\right) \mathbf{C}^{\mathrm{T}}\right) \quad\left(\text { Since } \mathrm{E}\left(\boldsymbol{\varepsilon}^{\boldsymbol{l}} \boldsymbol{\eta}^{\boldsymbol{I}^{\mathrm{T}}}\right)=0\right. \text {, as they are uncorrelated) } \\
& \mathrm{E}\left(\boldsymbol{\varphi}^{l^{\mathrm{T}}} \boldsymbol{\Psi}^{l}\right)=\boldsymbol{\Phi}^{l^{\mathrm{T}}} \mathbf{C} \boldsymbol{\Phi}_{s}^{l}+\operatorname{tr}\left(\mathrm{E}\left(\boldsymbol{\varepsilon}^{l} \boldsymbol{\varepsilon}_{s}^{l^{\mathrm{T}}}\right) \mathbf{C}^{\mathrm{T}}\right)
\end{aligned}
$$




\section{Appendix - C}

\section{C.1. $G^{l}$ when using SEREP modal expansion}

In case of SEREP expansion, $\mathbf{C} \boldsymbol{\Phi}_{s}^{l}=\boldsymbol{\Phi}^{l}$. Thus,

$$
\begin{aligned}
& \boldsymbol{\Phi}^{l^{\mathrm{T}}} \mathbf{C} \boldsymbol{\Phi}_{\boldsymbol{s}}^{l}=\boldsymbol{\Phi}^{\boldsymbol{l}^{\mathrm{T}}} \boldsymbol{\Phi}^{l}=\left\|\boldsymbol{\Phi}^{l}\right\|_{2}^{2} \\
& \boldsymbol{\Phi}_{\boldsymbol{s}}^{\boldsymbol{l}^{\mathrm{T}}} \mathbf{C}^{\mathrm{T}} \mathbf{C} \boldsymbol{\Phi}_{\boldsymbol{s}}^{\boldsymbol{l}}=\left(\mathbf{C} \boldsymbol{\Phi}_{\boldsymbol{s}}^{\boldsymbol{l}}\right)^{\mathrm{T}} \mathbf{C} \boldsymbol{\Phi}_{\boldsymbol{s}}^{\boldsymbol{l}}=\boldsymbol{\Phi}^{\boldsymbol{l}^{\mathrm{T}}} \boldsymbol{\Phi}^{\boldsymbol{l}}=\left\|\boldsymbol{\Phi}^{\boldsymbol{l}}\right\|_{2}{ }^{2}
\end{aligned}
$$

The mode shape matrix $\boldsymbol{\Phi}^{l}$ can be partitioned based on the measured and unmeasured dof as $\boldsymbol{\Phi}^{l^{T}}=\left[\begin{array}{ll}\boldsymbol{\Phi}_{s}^{l^{\mathrm{T}}} & \boldsymbol{\Phi}_{d}^{l^{\mathrm{T}}}\end{array}\right]$ where $\boldsymbol{\Phi}_{\boldsymbol{d}}^{l} \in \mathbb{R}^{d x 1}$ refers to the unmeasured $d o f$ and $s+d=n$.

Now from Eq. (4),

$$
\begin{aligned}
& \operatorname{tr}\left(\mathrm{E}\left(\boldsymbol{\varepsilon}^{\boldsymbol{l}} \boldsymbol{\varepsilon}_{\boldsymbol{s}}^{\boldsymbol{l}^{\mathrm{T}}}\right) \mathbf{C}^{\mathrm{T}}\right)=\operatorname{tr}\left(\left[\begin{array}{c}
\sigma_{\varepsilon}{ }^{2} \mathbf{I}_{\mathbf{s}} \\
\mathbf{0}
\end{array}\right] \mathbf{C}^{\mathrm{T}}\right) \text { where } \mathbf{O} \in \mathbb{R}^{d x s} \text { is a null matrix }
\end{aligned}
$$

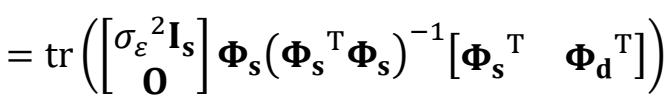

$$
\begin{aligned}
& =\sigma_{\varepsilon}{ }^{2} \operatorname{tr}\left(\boldsymbol{\Phi}_{\mathbf{s}}\left(\boldsymbol{\Phi}_{\mathbf{s}}^{\mathrm{T}} \boldsymbol{\Phi}_{\mathbf{s}}\right)^{-1} \boldsymbol{\Phi}_{\mathbf{s}}^{\mathrm{T}}\right)
\end{aligned}
$$

Since $\operatorname{tr}\left(\boldsymbol{\Phi}_{\mathbf{s}}\left(\boldsymbol{\Phi}_{\mathbf{s}}^{\mathrm{T}} \boldsymbol{\Phi}_{\mathbf{s}}\right)^{-1} \boldsymbol{\Phi}_{\mathbf{s}}^{\mathrm{T}}\right)=m$,

$$
\operatorname{tr}\left(\mathrm{E}\left(\boldsymbol{\varepsilon}^{l} \boldsymbol{\varepsilon}_{\boldsymbol{s}}^{\boldsymbol{l}^{\mathrm{T}}}\right) \mathbf{C}^{\mathrm{T}}\right)=\sigma_{\varepsilon}{ }^{2} m
$$

If all the sensor noises are assumed to be identical,

$$
\begin{aligned}
& \operatorname{tr}\left(\mathbf{C} \boldsymbol{\Sigma}_{\mathbf{N}}^{2} \mathbf{C}^{\mathrm{T}}\right)=\sigma_{\mathrm{N}}{ }^{2} \operatorname{tr}\left(\mathbf{C} \mathbf{C}^{\mathrm{T}}\right) \\
& =\sigma_{\mathrm{N}}{ }^{2} \operatorname{tr}\left(\boldsymbol{\Phi}\left(\boldsymbol{\Phi}_{\mathbf{s}}{ }^{\mathrm{T}} \boldsymbol{\Phi}_{\mathbf{s}}\right)^{-1} \boldsymbol{\Phi}_{\mathbf{s}}{ }^{\mathrm{T}} \cdot \boldsymbol{\Phi}_{\mathbf{s}}\left(\boldsymbol{\Phi}_{\mathbf{s}}{ }^{\mathrm{T}} \boldsymbol{\Phi}_{\mathbf{s}}\right)^{-1} \boldsymbol{\Phi}^{\mathrm{T}}\right) \\
& =\sigma_{\mathrm{N}}{ }^{2} \operatorname{tr}\left(\left[\begin{array}{l}
\boldsymbol{\Phi}_{\mathbf{s}} \\
\boldsymbol{\Phi}_{\mathbf{d}}
\end{array}\right]\left(\boldsymbol{\Phi}_{\mathbf{s}}{ }^{\mathrm{T}} \boldsymbol{\Phi}_{\mathbf{s}}\right)^{-1}\left[\begin{array}{ll}
\boldsymbol{\Phi}_{\mathbf{s}}^{\mathrm{T}} & \boldsymbol{\Phi}_{\mathbf{d}}^{\mathrm{T}}
\end{array}\right]\right) \\
& =\sigma_{\mathrm{N}}{ }^{2}\left(\operatorname{tr}\left(\boldsymbol{\Phi}_{\mathbf{s}}\left(\boldsymbol{\Phi}_{\mathbf{s}}{ }^{\mathrm{T}} \boldsymbol{\Phi}_{\mathbf{s}}\right)^{-1} \boldsymbol{\Phi}_{\mathbf{s}}{ }^{\mathrm{T}}\right)+\operatorname{tr}\left(\boldsymbol{\Phi}_{\mathbf{d}}\left(\boldsymbol{\Phi}_{\mathbf{s}}{ }^{\mathrm{T}} \boldsymbol{\Phi}_{\mathbf{s}}\right)^{-1} \boldsymbol{\Phi}_{\mathbf{d}}{ }^{\mathrm{T}}\right)\right) \\
& =\sigma_{\mathrm{N}}{ }^{2}\left(m+\operatorname{tr}\left(\boldsymbol{\Phi}_{\mathbf{d}}\left(\boldsymbol{\Phi}_{\mathbf{s}}{ }^{\mathrm{T}} \boldsymbol{\Phi}_{\mathbf{s}}\right)^{-1} \boldsymbol{\Phi}_{\mathbf{d}}{ }^{\mathrm{T}}\right)\right)
\end{aligned}
$$

Substituting Eq. (C.1.1-C.1.4) in Eq. (3),

$$
G^{l}=n{\sigma_{\varepsilon}}^{2}+\left\|\boldsymbol{\Phi}^{l}\right\|_{2}^{2}-\frac{\left(\left\|\boldsymbol{\Phi}^{l}\right\|_{2}^{2}+{\sigma_{\varepsilon}}^{2} m\right)^{2}}{{\sigma_{\mathrm{N}}}^{2}\left(m+\operatorname{tr}\left(\boldsymbol{\Phi}_{\mathbf{d}}\left(\boldsymbol{\Phi}_{\mathbf{s}}^{\mathrm{T}} \boldsymbol{\Phi}_{\mathbf{s}}\right)^{-1} \boldsymbol{\Phi}_{\mathbf{d}}^{\mathrm{T}}\right)\right)+\left\|\boldsymbol{\Phi}^{l}\right\|_{2}{ }^{2}}
$$




\section{References}

1. A.K. Pandey, M. Biswas, M.M. Samman, Damage detection from changes in curvature mode shapes, J. Sound Vib. 145 (1991) 321-332. doi:10.1016/0022-460X(91)90595-B.

2. Kondo, I., Hamamoto, T., 1996. Seismic Damage Detection of Multi-Story Buildings Using Vibration Monitoring, in: proc. of $11^{\text {th }}$ World Conference on Earthquake Engineering, 988, Acapulco, Mexico.

3. F. Pelayo, A. Skafte, M.L. Aenlle, R. Brincker, Modal Analysis Based Stress Estimation for Structural Elements Subjected to Operational Dynamic Loadings, Exp. Mech. 55 (2015) 1791-1802. doi:10.1007/s11340-015-0073-6.

4. M. Tarp $\varnothing$, B. Nabuco, A. Skafte, J. Kristoffersen, J. Vestermark, S. Amador, R. Brincker, Operational modal analysis based prediction of actual stress in an offshore structural model, in: proc. of 10th International Conference on Structural Dynamics. (2017) 2262-2267, Rome, Italy. doi:10.1016/j.proeng.2017.09.234.

5. M. Tarp $\varnothing$, B. Nabuco, C. Georgakis, R. Brincker, Expansion of experimental mode shape from operational modal analysis and virtual sensing for fatigue analysis using the modal expansion method, Int. J. Fatigue. 130 (2020) 105280. doi:doi.org/10.1016/j.ijfatigue.2019.105280.

6. C. Papadimitriou, C.P. Fritzen, P. Kraemer, E. Ntotsios, Fatigue predictions in entire body of metallic structures from a limited number of vibration sensors using Kalman filtering, Struct. Control Heal. Monit. 18 (2011) 554573. doi:10.1002/stc.395.

7. Dertimanis, V.K., Chatzi, E.N., Eftekhar Azam, S., Papadimitriou, C., 2016. Fatigue assessment in steel railway bridges using output only vibration measurements and partial structural information, in: proc. of Third International Conference on Railway Technology: Research, Development and Maintenance, 139, Sardinia, Italy. doi:10.4203/ccp.110.139.

8. P.C. Shah, F.E. Udwadia, A Methodology for optimal sensor locations for identification of dynamic, systems, J. Appl. Mech. 45 (1978) 188-196. doi:10.1115/1.3424225.

9. F.E. Udwadia, Methodology for Optimum Sensor Locations for Parameter Identification in Dynamic Systems, J. Eng. Mech. 120 (1994). doi:10.1061/(asce)0733-9399(1994)120:2(368).

10. D.C. Kammer, Sensor placement for on-orbit modal identification and correlation of large space structures, J. Guid. Control. Dyn. 14 (1991) 251-259. doi:10.2514/3.20635.

11. D.C. Kammer, Effects of noise on sensor placement for on-orbit modal identification of large space structures, J. Dyn. Syst. Meas. Control. 114 (1992) 436-443. doi:10.2514/6.1993-1704.

12. D.C. Kammer, Effect of model error on sensor placement for on-orbit modal identification of large space structures, J. Guid. Control. Dyn. 15 (1992) 334-341. doi:10.2514/3.20841.

13. M.I. Friswell, J.E. Mottershead, Finite element model updating in structural dynamics, Springer-Science and Business Media., 2013. doi:10.1007/978-94-015-8508-8.

14. C. Schedlinski, M. Link, An Approach to Optimal Pick-up and Exciter Placement, in: proc. of 14th International Modal Analysis Conference. (1996) 376-382, Michigan, United States.

15. G. Heo, M.L. Wang, D. Satpathi, Optimal transducer placement for health monitoring of long span bridge, Soil Dyn. Earthq. Eng. 16 (1997) 495-502. doi:10.1016/S0267-7261(97)00010-9.

16. E.M. Hernandez, Efficient sensor placement for state estimation in structural dynamics, Mech. Syst. Signal Process. 85 (2017) 789-800. doi:10.1016/j.ymssp.2016.09.005.

17. S. Zhu, X.-H. Zhang, Y.-L. Xu, S. Zhan, Multi-Type Sensor Placement for Multi-Scale Response Reconstruction, Adv. Struct. Eng. 16 (2013). doi:10.1260/1369-4332.16.10.1779. 
18. Y.L. Xu, X.H. Zhang, S. Zhu, S. Zhan, Multi-type sensor placement and response reconstruction for structural health monitoring of long-span suspension bridges, Sci. Bull. 61 (2016) 313-329. doi:10.1007/s11434-016-10007.

19. C. Papadimitriou, Y. Haralampidis, K. Sobczyk, Optimal experimental design in stochastic structural dynamics, Probabilistic Eng. Mech. 20 (2005) 67-78. doi:10.1016/j.probengmech.2004.06.002.

20. X.H. Zhang, S. Zhu, Y.L. Xu, X.J. Homg, Integrated optimal placement of displacement transducers and strain gauges for better estimation of structural response, Int. J. Struct. Stab. Dyn. 11 (2011) 581-602. doi:10.1142/s0219455411004221.

21. R. Soman, T. Onoufriou, M.A. Kyriakides, R.A. Votsis, C.Z. Chrysostomou, Multi-type, multi-sensor placement optimization for structural health monitoring of long span bridges, Smart Struct. Syst. 14(1) (2014) 55-70. doi:10.12989/sss.2014.14.1.055.

22. L. Dongsheng, Sensor placement methods and evaluation criteria in structural health monitoring, Ph.D. Thesis, Universität Siegen, 2012.

23. Y. Ting-Hua, L. Hong-Nan, Methodology developments in sensor placement for health monitoring of civil infrastructures, Int. J. Distrib. Sens. Networks. 8 (2012). doi:10.1155/2012/612726.

24. V. Mallardo, M.H. Aliabadi, Optimal sensor placement for structural, damage and impact identification: A review, SDHM Struct. Durab. Heal. Monit. 9 (2013) 287-323. doi:10.1007/s10336-016-1361-3.

25. G.F. Gomes, S.S. da Cunha, P. da Silva Lopes Alexandrino, B. Silva de Sousa, A.C. Ancelotti, Sensor placement optimization applied to laminated composite plates under vibration, Struct. Multidiscip. Optim. 58 (2018) 20992118. doi:10.1007/s00158-018-2024-1.

26. W. Ostachowicz, R. Soman, P. Malinowski, Optimization of sensor placement for structural health monitoring: a review, Struct. Heal. Monit. 18 (2019) 963-988. doi:10.1177/1475921719825601.

27. T.G. Carne, C.R. Dohrmann, A modal test design strategy for model correlation, in: proc. of $13^{\text {th }}$ International Modal Analysis Conference. (1995) 927-933, Tennessee, United States.

28. R.J. Allemang, D.L. Brown, A correlation coefficient for modal vector analysis, in: proc. of First International Modal Analysis Conference. (1982) 110-116, Florida, United States.

29. Lenticchia, E., Ceravolo, R., Antonaci, P., 2018. Sensor Placement Strategies for the Seismic Monitoring of Complex Vaulted Structures of the Modern Architectural Heritage. Shock Vib. 3739690. doi:10.1155/2018/3739690.

30. T.H. Yi, H.N. Li, M. Gu, Optimal sensor placement for structural health monitoring based on multiple optimization strategies, Struct. Des. Tall Spec. Build. 20 (2011) 881-900. doi:10.1002/tal.712.

31. G.F. Gomes, F.A. de Almeida, P. da Silva Lopes Alexandrino, S.S. da Cunha, B.S. de Sousa, A.C. Ancelotti, A multiobjective sensor placement optimization for SHM systems considering Fisher information matrix and mode shape interpolation, Eng. Comput. 35 (2019) 519-535. doi:10.1007/s00366-018-0613-7.

32. K.H. Tong, N. Bakhary, A.B.H. Kueh, A.Y. Mohd Yassin, Optimal sensor placement for mode shapes using improved simulated annealing, Smart Struct. Syst. 13 (2014) 389-406. doi:10.12989/sss.2014.13.3.389.

33. M. Meo, G. Zumpano, On the optimal sensor placement techniques for a bridge structure, Eng. Struct. 27 (2005) 1488-1497. doi:10.1016/j.engstruct.2005.03.015.

34. Murugan Jaya, M., Ceravolo, R., Matta, E., Zanotti Fragonara, L., 2018. Performance of Sensor Placement Strategies Used in System Identification Based on Modal Expansion, in: proc. of 9th European Workshop on Structural Health Monitoring, EWSHM-0080, Manchester, United Kingdom. 
35. E. Reynders, K. Maes, G. Lombaert, G. De Roeck, Uncertainty quantification in operational modal analysis with stochastic subspace identification: Validation and applications, Mech. Syst. Signal Process. 66-67 (2016) 13-30. doi:10.1016/j.ymssp.2015.04.018.

36. E. Reynders, R. Pintelon, G. De Roeck, Uncertainty bounds on modal parameters obtained from stochastic subspace identification, Mech. Syst. Signal Process. 22 (2008) 948-969. doi:10.1016/j.ymssp.2007.10.009.

37. M. Döhler, X.B. Lam, L. Mevel, Uncertainty quantification for modal parameters from stochastic subspace identification on multi-setup measurements, Mech. Syst. Signal Process. 36 (2013) 562-581. doi:10.1016/j.ymssp.2012.11.011.

38. M. Döhler, F. Hille, X.B. Lam, L. Mevel, W. Rücker, Confidence intervals of modal parameters during progressive damage test, in: Link. Model. Exp. Vol. 2, Springer, New York, NY, 2011: pp. 237-250. doi:10.1007/978-1-44199305-2_17.

39. G. Tondreau, E. Reynders, A. Deraemaeker, Towards a more realistic modelling of the uncertainty on identified mode shapes due to measurement noise, J. P 305 (2011) 012002. doi:10.1088/1742-6596/305/1/012002.

40. R.J. Guyan, Reduction of stiffness and mass matrices, AIAA J. 3 (1965) 380-380. doi:10.2514/3.2874.

41. L.F.F. Miguel, R.C.R. de Menezes, L.F.F. Miguel, Mode Shape Expansion from Data-Based System Identification Procedures, Meconial Comput. 25 (2006) 1593-1602.

42. K. Robert L, Reduction of structural frequency equations., AIAA J. 11 (1973) 892-892. doi:10.2514/3.6852.

43. J. O'Callahan, P. Avitabile, R. Riemer, System equivalent reduction expansion process (SEREP), in: proc. of $7^{\text {th }}$ International Modal Analysis Conference. (1989) 29-37, Las Vegas, United States.

44. J. O'Callahan, L. Ping, A Non-Smoothing SEREP Process for Modal Expansion, in: proc. of $12^{\text {th }}$ International Modal Analysis Conference. (1994) 232-238, Honolulu, Hawaii.

45. L. Yao, W.A. Sethares, D.C. Kammer, Sensor placement for on-orbit modal identification via a genetic algorithm, AIAA J. 31 (1993). doi:10.2514/3.11868.

46. C. Papadimitriou, Pareto optimal sensor locations for structural identification, Comput. Methods Appl. Mech. Eng. 194 (2005) 1655-1673. doi:10.1016/j.cma.2004.06.043.

47. Yi, T.H., Li, H.N., Gu, M., 2011. Optimal sensor placement for health monitoring of high-rise structure based on genetic algorithm. Math. Probl. Eng. 395101. doi:10.1155/2011/395101.

48. Soman, R., Malinowski, P., 2019. A Real-Valued Genetic Algorithm for Optimization of Sensor Placement for Guided Wave-Based Structural Health Monitoring. J. Sensors. 9614630. doi:10.1155/2019/9614630.

49. R. Soman, P. Kudela, K. Balasubramaniam, S.K. Singh, P. Malinowski, A study of sensor placement optimization problem for guided wave-based damage detection, Sensors (Basel). 19(8) (2019). doi:10.3390/s19081856.

50. J.H. Holland, Adaptation in Natural and Artificial systems, University of Michigan Press, Ann Arbor, 1975.

51. K.A. De-Jong, An Analysis of the Behavior of a Class of Genetic Adaptive Systems, Ph.D. Thesis, University of Michigan, 1975.

52. D. E Goldberg, Genetic Algorithms in Search, Optimization and Machine Learning, 1st ed., Addison-Wesley Publishing Company, 1989.

53. H. Randy L, H. Sue Ellen, Practical genetic algorithms, 2nd ed., John Wiley \& Sons, Inc., Hoboken, New Jersey, 1998.

54. Global Optimization Toolbox User's Guide R2018b, The MathWorks, Inc. 3 Apple Hill Drive Natick, MA 017602098, 2018. https://www.mathworks.com/help/pdf_doc/optim/optim_tb.pdf.

55. K. Deep, K.P. Singh, M.L. Kansal, C. Mohan, A real coded genetic algorithm for solving integer and mixed integer optimization problems, Appl. Math. Comput. 212 (2009) 505-518. doi:10.1016/j.amc.2009.02.044. 
56. K. Deb, An efficient constraint handling method for genetic algorithms, Comput. Methods Appl. Mech. Eng. 186 (2000) 311-338. doi:10.1016/S0045-7825(99)00389-8.

57. R. Brincker, C.E. Ventura, Introduction to Operational Modal Analysis, 1st ed., John Wiley \& Sons Ltd, West Sussex, United Kingdom, 2015. doi:10.1002/9781118535141.

58. H.Y. Guo, L. Zhang, L.L. Zhang, J.X. Zhou, Optimal placement of sensors for structural health monitoring using improved genetic algorithms, Smart Mater. Struct. 13 (2004) 528-534. doi:10.1088/0964-1726/13/3/011. 


\section{An optimal sensor placement strategy for reliable expansion of mode shapes under measurement noise and modelling error}

Jaya, Mahesh Murugan

Elsevier

Jaya MM, Ceravolo R, Zanotti Fragonara L, Matta E. (2020) An optimal sensor placement strategy for reliable expansion of mode shapes under measurement noise and modelling error. Journal of Sound and Vibration, Volume 487, November 2020, Article number 115511

https://doi.org/10.1016/j.jsv.2020.115511

Downloaded from Cranfield Library Services E-Repository 